\title{
Analysis of the radar reflectivity of aircraft vortex wakes
}

\author{
By KARIM SHARIF ${ }^{1}$ AND ALAN WRAY \\ 'NASA Ames Research Center, Moffett Field, CA 94035, USA
}

(Received ?? and in revised form ??)

Radar has been proposed as a way to track wake vortices to reduce aircraft spacing and tests have revealed radar echoes from aircraft wakes in clear air. The results are always interpreted qualitatively using Tatarski's theory of weak scattering by isotropic atmospheric turbulence. The goal of the present work was to predict the value of the radar cross-section (RCS) using simpler models. This is accomplished in two steps. (1) First, the refractive index is obtained. Since the structure of aircraft wakes is different from atmospheric turbulence, three simple mechanisms specific to vortex wakes are considered: (A) Radial density gradient in a 2D vortex (B) 3D fuctuations in the vortex cores (C) Adiabatic transport of atmospheric fluid in a $2 \mathrm{D}$ oval surrounding the pair of vortices. The index of refraction is obtained more precisely for the $2 \mathrm{D}$ mechanisms than for the 3D ones. (2) In the second step, knowing the index of refraction, a scattering analysis is performed. Tatarski's weak scattering approximation is kept but the usual assumptiuns of a far-field and a uniform incident wave are dropped. Neither assumption is generally valid for a wake that is coherent across the radar beam. For analytical insight, a simpler approximation that invokes, in addition to weak scattering, the far-field and wide cylindrical beam assumptions, is also developed and compared with the more general analysis. The predicted RCS values for the oval surrounding the vortices (mechanism C) agree with the experiments of Gilson (1992) conducted over a wide range of frequencies. However, the predictions have a cut-off away from normal incidence which is not present in the measurements. Estimates suggest that this is due to turbulence in the baroclinic vorticity generated at the boundary of the oval. The reflectivity of a vortex itself (mechanism A) is comparable to that of the oval (mechanism C) but cuts-off at frequencies lower than those considered in all the experiments to date. The RCS of a vortex happens to peak at the frequency (about $49 \mathrm{MHz}$ ) where atmospheric radars (known as ST radars) operate and so the present prediction could be verified in the future. Finally, we suggest that hot engine exhaust could increase ?CS by $40 \mathrm{db}$ and reveal vortex circulation, provided its mixing with the surroundings is prevented in the laminarising flow of the vortices.

\section{Introduction}

\subsection{Motivation}

The photographs of fluid motion in Van Dyke's (1982) album are all made possible by an optical effect in the visual range of the electromagnetic spectrum, for instance light scattering by smoke and the schlieren method. Fluid motions can also be "visible" to radio waves: an article by Ottersten (1969) displays remarkable photographs of atmospheric vortices imaged by radar in clear air. These include bouyant vortex rings, roll cells aligned with the wind in a stably stratified layer, and kelvin-Helmholtz rollers in the tropopause. Perhaps soneday we will see radar photographs of aircraft wakes as well. 
An important practical problem is that the vortex wakes of large aircraft pose a hazard to following aircraft and therefore, during instrument take-offs and landings, fixed distances are maintained between aircraft. These spacings are thought to be too conservative most of the time and to increase airport capacity, NASA has set itself the goal of developing a system which lynamically sets the spacing (Perry et al.1997). Several elements comprise this system including computer simulation of wakes and vortex detection from the ground.

In the NASA effort, two methods of vortex detection are being concentrated upon: doppler lidar at infrared frquencies and radar. Lidar is the more mature of the two efforts and relies on scattering by aerosols such as water droplets which may begin to form in the low pressure of the vortices even when cloud is not visiblet. Field tests at airports using $10.6 \mu \mathrm{m}$ lidar (Campbell et al.1997, Köpp 1994) have detected vortices at ranges of up to $300 \mathrm{~m}$; in clear weather this limit is set by the depth of focus of the optics. Hannon \& Thomson (1994) report ranges of up to $4 \mathrm{~km}$ at $2.09 \mu \mathrm{m}$ using high energy pulsed lidar.

There are two motivations for considering radar. First is the concern that lidar cannot work in fog and rain due to increased absorption. Radar, on the other hand, is unaffected by rain and fog at sufficiently low frequencies. The second factor is that while lidar has adequate range to protect the modestly sized approach corridor currently set by the planners, radar has a potentially greater range.

\subsection{Previous theoretical work}

The results of radar tests that have detected aircraft wakes are all interpreted using the theory (Tatarski 1961) of scattering by refractive index fluctuations caused by atmospheric motions in clear air. The theory is based on the fact that a turbulent velocity field in the presence of mean vertical gradients of potential temperature and humidity lead to fluctuations in refractive index. The velocity field is assumed to be statistically homogeneous and isotropic locally and to lie in the Kolmogorov inertial range. Refractive index gradients are therefore also homogeneous and isotropic. One result of the analysis is that if the wave number of the incident radio wave is $k$, the only wavevector of the refractive index capable of scattering to the observer is:

$$
\mathbf{k}=k(\widehat{\mathbf{i}}-\widehat{\mathbf{o}}) \text {, }
$$

where $\widehat{\mathbf{i}}$ and $\hat{\mathbf{o}}$ are the unit vectors in the incident wave direction and the direction from the target to the observer, respectively. This is the so-called Bragg condition. In particular for backscattering $(\widehat{o}=-\widehat{\mathbf{i}})$ one gets:

$$
\mathbf{k}=2 \widehat{k \mathbf{i}}
$$

Thus, the only flow wavevector which backscatters is aligned with the radar beam and has twice the radar wavenumber. According to Tatarski's theory the radar cross-section per unit volume of isotropic turbulence in the inertial range is (e.g. see Ottersten 1969)

$$
\eta=0.38 C_{n}^{2} \lambda^{-1 / 3}
$$

+ While scattering is the usually proffered explanation, it is interesting to note that according to Carlon (1970) water aerosols are capable of strong absorption and emission in the 8-13 $\mu$ range and that "water droplets in this wavelength region behave much more as absorbers and emitters and much less as Mie scatterers that has been imagined." Two of three vortex lidar tests were $10.6 \mu$ and therefore re-emission could very well be present. This fact suggests that passive radiometry of condensed water aerosol in the vortex cores might be another method of detection. 
where $\lambda$ is the radar wavelength and $\eta$ has units of $L^{-1}$. The radar cross-section can thus be obtained if one knows $C_{n}^{2}$, the so-called second-order structure constant of the index of refraction fluctuations. Equation (1.3) has become widely quoted in the aircraft vortex detection context as well, with the belief that aircraft wake turbulence creates a turbulent energy cascade similar to atmospheric turbulence. Equation (1.3) is also used to reduce experimental data (Chadwick et al., Nespor et al.1994). In particular, the raw radar cross-section (RCS) reported by the radar is divided by the volume of the pulse to obtain $\eta$ and then the value of $C_{n}^{2}$, calculated using (1.3), is plotted.

Marshall \& Myers (1996) attempted to predict $C_{n}^{2}$ as a function of position in a Reynolds averaged calculation of a statistically $2 \mathrm{D}$ vortex wake by using an algebraic closure relation for $C_{n}^{2}$ in the inertial range in terms of gradients of mean velocity and potential refractive index. This model is analogous to that given by Ottersten (1969, p. 1184) for atmospheric turbulence.

There are also classified reports (with unclassified titles) cited by Gilson (1992) to which we were not privy.

\subsection{Previous experiments}

(i) Noonkester \& Richter (1980) detected the wakes of aircraft taking off ( $f=3 \mathrm{GHz}$, $\lambda=10 \mathrm{~cm}$, range $R=100-300 \mathrm{~m})$. Their FM-CW radar, in contrast to a pulse radar, transmits and receives continuously allowing for a small minimum range. An interesting sinusoidal pattern was observed in the trace of target height (above a fixed ground location) vs. time. They interpreted this to be hot engine exhaust spiraling around each vortex. Whether engine exhaust might be put to use for vortex detection during approach is briefly discussed in $\S 6.2$.

(ii) Using a similar radar, Chadwick et al.(1984) detected the wakes of both departing and arriving aircraft but do not distinguish between the two in their results. The radar used was rather modest ( $200 \mathrm{~W}$ of power, $8 \mathrm{ft}$ diameter dish) and the ranges were $R \leqslant 1$ $\mathrm{km}$. The distribution functions of RCS in the atmosphere ahead of the plane and the wake behind the plane overlapped a little and for positive identification of the wake, the authors suggested use of the spread in doppler velocity. They suggested two mechanisms for scattering. First, they argued that aircraft vortices created a turbulent cascade similar to those assumed in the theory of atmospheric scattering (Tatarski 1961) but with a $C_{n}^{2}$ higher than that of the ambient atmosphere. They found no directional sensitivity which lent some credibility to the the assumption of isotropy. Secondly, they suggested that heat and moisture from the engine further increases reflectivity.

(iii) Using a $1 \mathrm{MW}$ pulse doppler radar $(f=5.6 \mathrm{GHz}, R=2.7 \mathrm{~km})$, Nespor et al.(1994) detected the vortices of a small fighter aircraft in approach configuration looking axially along the vortex.

(iv) So far, Gilson (1992) has the best documented and controlled experiment. Using powerful pulse doppler radars having $2-7 \mathrm{MW}$ of peak power, he measured the radar cross-sections of the wake of a C-5 A aircraft at a range of $15 \mathrm{~km}$. Returns were detectable at five frequencies between $0.162 \mathrm{G} \mathrm{Hz}$ and $5.7 \mathrm{GHz}$ but not at $35 \mathrm{GHz}$. From the observation that the radar cross-section was relatively flat as a function of frequency and dropped off somewhere above $f=5.7 \mathrm{GHz}$, he concluded that particulates were not involved (they would give $f^{4}$ Rayleigh scattering). He noted that the frequency dependence was not the Kolmogorov $f^{1 / 3}$. The RCS decreased with altitude and vanished above the tropopause from which he concluded that the scattering mechanism was related to "low altitude climate." Fluid mechanicians will anticipate that the relevant fact here is the decrease with height of the Brünt-Väisälä frequency, the appropriate measure of stratification. He found that the RCS (measured $1 \mathrm{~km}$ behind the plane) was insensitive to engine thrust 
and the RCS (measured at 10,000 altitude and $0.6 \mathrm{~km}$ behind the plane) was insensitive to Hap setting. He thus concluded that the main mechanism was "turbulent mixing by the wake vortices of existing atmospheric index of refraction gradients."

(v) For completeness, we mention a test (lannuzzelli et al.1998) in which a radar was used to detect index of refraction variations caused, remarkably, by sound waves launched as a tracer into the aircraft wake from the ground. The sound waves are scattered by the vortex flow and the doppler shift they induce on a radar beam can be measured. This technique is an extension of a routinely used method for wind profiling and is known as RASS (Radio Acoustic Sounding System).

\subsection{Present work}

Led by the observation that turbulence is suppressed in an aircraft vortex, $\S 2$ considers three simple laminar and largely two dimensional mechanisms for producing refractive index variations.

Given the index of refraction, $\S 3$ performs a scattering analysis using the Born weak scattering approximation. It is argued that the usual assumptions of a uniform incident wave and a far-field are not generally valid for a long and coherent target such as an aircraft wake. Therefore the shape of the incident beam and full Green's function are taken into account. This analysis has to be implemented numerically and so to provide a simple analytical result, and for insight into the results, a simplified analysis is presented in $\S 4$. This analysis makes the approximations of a far-field and a cylindrical beam that is wider than the cross-sectional width of the target.

Section 5 presents numerical predictions of the radar cross-section for the $2 \mathrm{D}$ mechanisms, comparing the approximate analysis, the full Born analysis and experiment. Section 6 presents some practical reccomendations including a sample design for an ST type radar to detect the vortices themselves.

\section{Three mechanisms for inhomogeneities in dielectric constant}

The three sub-subsections which follow discuss the change in dielectric constant due to three effects: (i) radial density gradient in the core of each vortex, (ii) three dimensional instabilities in the vortex cores, and, (iii) transport by the vortices of the atmospheric density. Following Gilson (1994) we assume that particulates are unimportant to the scattering mechanism in clear air.

The refractive index $n$ of humid air for frequencies below $20 \mathrm{GHz}$ is given by Thayer (1974):

$$
(n-1) \times 10^{6}=77.6\left(\frac{p_{a}}{T}\right)+64.8\left(\frac{p_{v}}{T}\right)+3.776 \times 10^{5}\left(\frac{p_{v}}{T^{2}}\right) .
$$

Here $p_{a}$ is the partial pressure $(\mathrm{mb})$ of dry air, $p_{v}$ is the partial pressure (mb) of water vapor and $T$ is the temperature $(\mathrm{K})$. The first two terms are due to the induced polarization of air and water molecules, respectively. The third term is due to the permanent dipole moment of the water vapor molecule. $p_{v}$ can be obtained from the relative humidity, RH $\equiv p_{v} / p_{s a t}$, where $p_{s a t}$ is the saturation pressure of water vapor and can be obtained as a function of temperature using the formula in Reid et al.(1987, p. 757). The dielectric constant is simply $\varepsilon=n^{2}$. 
2.1. Radial density gradient in the vortex cores

The radial momentum equation (for laminar flow) together with the assumption of homentropic flow can be integrated to yield the radial density variation in each vortex:

$$
\frac{\rho(r)}{\rho_{\infty}}=\left(1-(\gamma-1) \int_{r}^{\infty} \frac{1}{r} \frac{V_{\theta}^{2}}{c_{\infty}^{2}} d r\right)^{\frac{1}{r-1}} .
$$

Here $c_{\infty}$ and $\rho_{\infty}$ are the ambient speed of sound and density, respectively. Equation (2.2) applies to both the density of water vapor and the density of air.

Two profiles for the circumferential velocity, $V_{\theta}$ were considered. The first is that of the Lamb-Oseen vortex:

$$
V_{\theta}=\frac{\Gamma}{2 \pi r}\left(1-\mathrm{e}^{-r^{2} / \delta^{2}}\right)
$$

The following values, provided by Rennich (1997) as representative of a large commercial aircraft were used: core radius, $r_{0}=4.724 \mathrm{~m}$ and circulation, $\Gamma=526 \mathrm{~m}^{2} \mathrm{~s}^{-1}$. We take the core radius, $r_{0}$, to be the location where the tangential velocity has a peak and for the Lamb-Oseen profile we have $\delta=r_{0} / 1.12141$. With these values Eqs. (2.2) and (2.3) give a small density dip: $\rho(0) / \rho_{\infty}=.998$.

The second profile of circumferential velocity was provided to us by Dr. P. Spalart of Boeing and represents his fit to flight observations:

$$
V_{\theta}=\frac{\Gamma}{2 \pi \eta b_{0}} \begin{cases}1189 \eta^{2}, & \text { for } \eta<.0103 \\ {\left[(1.27+\log (\eta) / 4)^{-14}+1\right]^{-1 / 14},} & \text { otherwise }\end{cases}
$$

Here

$$
\eta \equiv \frac{r}{b_{0}}
$$

We chose $b_{0}=47.88 \mathrm{~m}$, the value of vortex spacing provided by Rennich (1997). This profile gives a greater minimum: $\rho(0) / \rho_{\infty}=0.988$. Figure 1 plots the relative change

$$
\Delta \varepsilon_{\mathbf{r}} \equiv \frac{\varepsilon}{\varepsilon_{0}}-1
$$

in dielectric constant from the ambient value $\left(\varepsilon_{0}\right)$ for the two velocity profiles and for two cases of ambient relative humidity (dry and $80 \%$ ). The ambient temperature and pressure were taken to be $15^{\circ} \mathrm{C}$ and $1013.25 \mathrm{mb}$, respectively. We observe that humidity has a weak influence and the profile of Spalart has a more pronounced dip of $\Delta \varepsilon_{\mathrm{r}}$ at the vortex axis $\approx-6 \times 10^{-6}$.

\subsection{Three dimensional fluctuations in the vortex cores .}

Little is known about turbulence within an aircraft trailing vortex but it has come to be appreciated that both the cascade to small scales and turbulence production are drastically reduced by rotation. Turbulence models currently used in aerodynamic as well as atmospheric codes are unable to capture these effects.

With respect to the first effect, the rate of energy transfer and hence dissipation is reduced (see, for example, Bardina et al.1985) because rotation scrambles non-linear interactions.

A more important effect is the drastic alteration of turbulence production. There are three mean velocity gradients which can act as turbulence production mechanisms within the vortex:

(i) There is a jet/wake flow along the vortex axis but this decays quite rapidly behind the wing. 


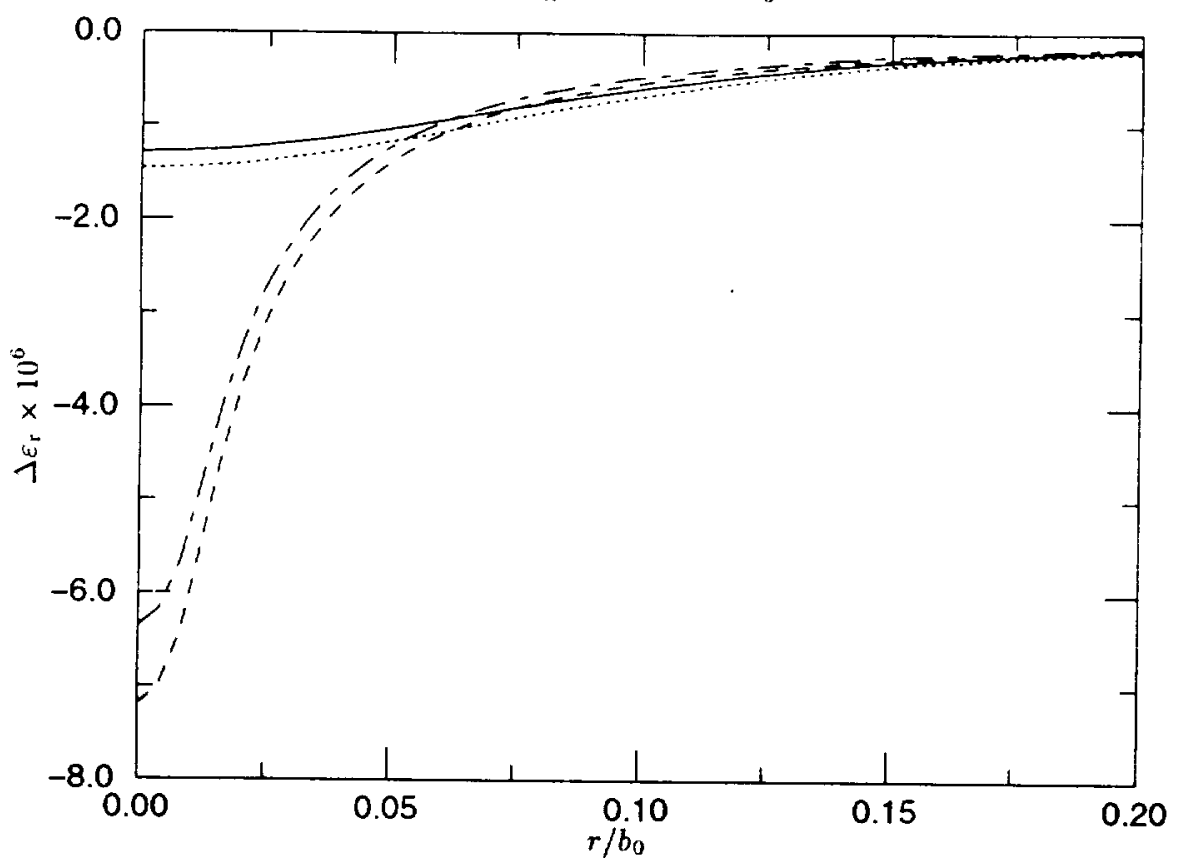

FIGURE 1. Radial distribution of $\Delta \varepsilon_{\mathrm{r}}$ inside a vortex. - - Lamb-Oseen vortex, $(\mathrm{RH})_{\infty}=0 \%$; $\cdots \cdots$ : Lamb-Oseen vortex, (RH) $)_{\infty}=80 \%$; - - : Spalart's profile, (Eq. 2.4), (RH) $)_{\infty}=0 \%$; - Spalart's profile, (Eq. 2.4), $(\mathrm{RH})_{\infty}=80 \%$.

(ii) There is a circumferential velocity around the axis. In the central part of the core the production of turbulent kinetic energy is zero because of solid body rotation. As one moves away from the center, fluid elements are sheared as they rotate. The Bradshaw criterion for stability of shear in a rotating frame when applied to a vortex with single sign vorticity predicts that the flow is locally stable at each $r$ (LeBlanc \& Cambon 1997). Indeed Qin (1998) found via direct numerical simulation of turbulence within the vortex that there is negative production of turbulent kinetic energy.

(iii) Finally, the only remaining mechanism for turbulence production is the strain induced by one vortex upon the other. It is responsible for the commonly observed Crow (1970) instability which has a wavelength of about nine times the vortex separation. A shorter wavelength instability, called the Widnall instability in the context of vortex rings is also possible at sufficiently high Reynolds numbers. This instability has been observed in the laboratory by Thomas \& Auerbach (1994) and Leweke \& Williamson (1998).

The question arises whether even shorter waves can be excited by the strain in the presence of the stabilizing effect of the rotating shear. To address this question without performing a full stability analysis of a vortex pair we consider a stability analysis which accounts for only the local velocity gradient. Such an analysis is valid for waves much smaller than the vortex core size. The local velocity gradient following a fluid element has three parts: rotation, strain induced by the other vortex and rotating shear. The first two alone lead to the elliptic instability (e.g. Landman \& Saffman 1987). To this we add rotating shear. The velocity gradient matrix then becomes:

$$
A=\left(\begin{array}{ccc}
0 & -\gamma-e & 0 \\
\gamma-e & 0 & 0 \\
0 & 0 & 0
\end{array}\right)+S\left(\begin{array}{ccc}
-\sin \Omega t \cos \Omega t & \cos ^{2} \Omega t & 0 \\
-\sin ^{2} \Omega t & \sin \Omega t \cos \Omega t & 0 \\
0 & 0 & 0
\end{array}\right) .
$$

The first term represents the elliptic streamline flow which has rotation and strain while 


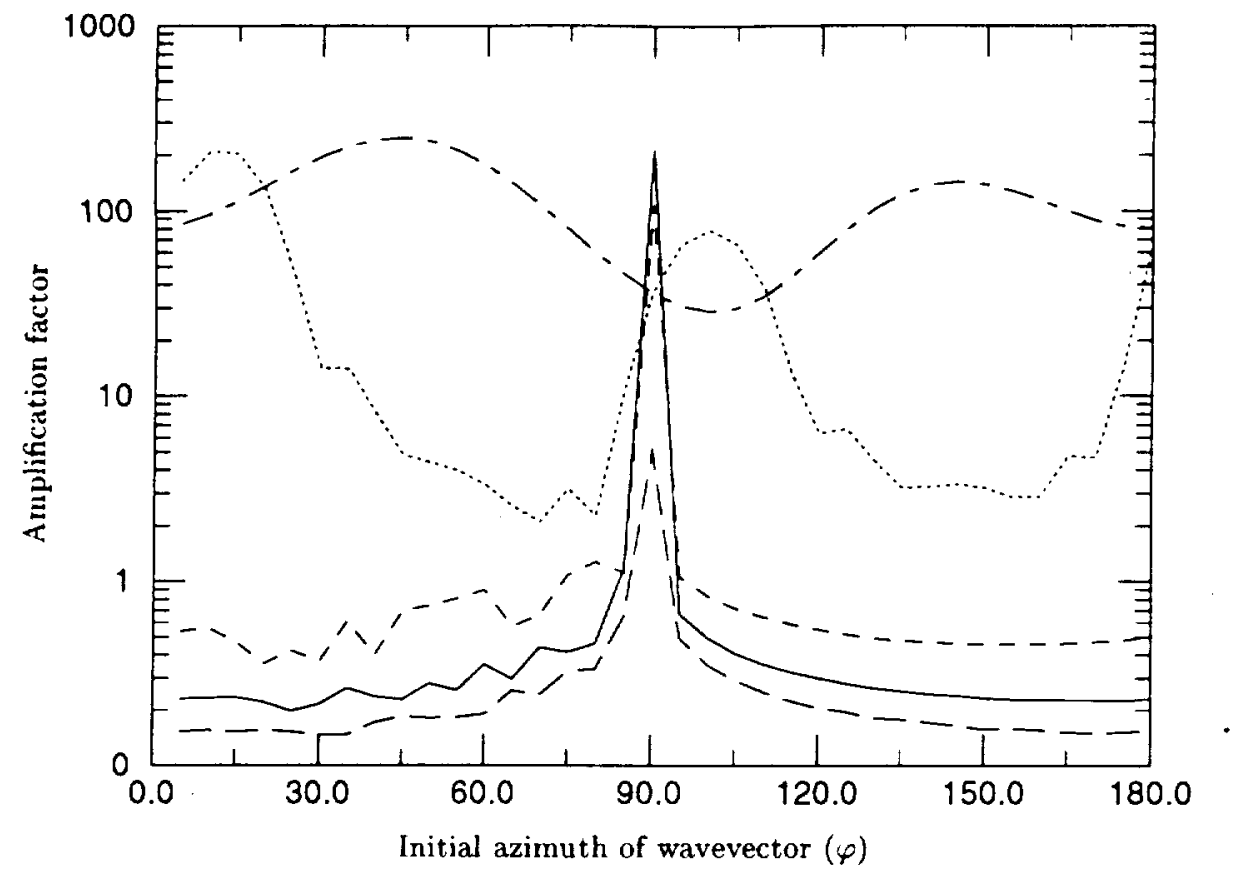

Figure 2. Amplification factor of disturbances at various locations in a Lamb-Oseen vortex subject to strain by another vortex - - : $r / \delta=0.0 ; \cdots \cdots \cdots: r / \delta=0.01 ;-\cdots-r / \delta=0.10$; $\longrightarrow: r / \delta=0.20 ;---: r / \delta=030$.

the second term represents a shear (of rate $S$ ) rotating with angular velocity $\Omega$. The value of $\Omega$ was chosen to be the rate of rotation of fluid particles in the elliptic flow, i.e. $\Omega=\left(\gamma^{2}-\epsilon^{2}\right)^{1 / 2}$. Had $\Omega$ been chosen to be the rate of rotation, $\gamma$, of fluid particles without the strain then the shear axes would get ahead of the axes of a fluid element which is not physical. For an axisymmetric circumferential flow $u_{\theta}(r)$ we have

$$
\gamma=\frac{u_{\theta}}{r} \text { and } S=-\left(\frac{\partial u_{\theta}}{\partial r}-\frac{u_{\theta}}{r}\right)
$$

The strain rate due to the other member of a vortex pair is

$$
e=2 \times \frac{\Gamma}{2 \pi b_{0}^{2}}
$$

where the induced strain has been multiplied by 2 in order that the growth ate and ellipticity of the elliptic streamline flow match that of a vortex of uniform vorticity surrounded by potential flow. The evolution of a single mode was considered at several values of $r / \delta$ inside a Lamb-Oseen vortex with a value of $\delta / b_{0}=.088$ given by Rennich (1997) for a large aircraft in approach. The modes investigated were those known to be the most unstable for the elliptic streamlini flow. These modes have wavevector lying in a cone at $60^{\circ}$ relative to the vortex axis. Within this cone the initial azimuthal angle $\varphi$ is free. Figure 2 shows the amplification factor of the velocity perturbation during the time that the vortex pair descends five vortex spacings. The different curves are for different locations, $r / \delta$ within the core. At the center of the core (chain dashed line) there is no shear and the elliptic instability is active for all $\varphi$. As one moves outward, the shear stabilizes all modes except those located in a thin band near $\varphi=90^{\circ}$. This wavevector direction corresponds wavefronts that are parallel to the shear flow and therefore not deformed 
by it. By $r / \delta=0.3$ (long-lashed curve) the growth of even these waves is suppressed by the shear. We thus conclude that roughly only the inner $20 \%$ of the vortex core, or a diameter of $170 \mathrm{~cm}$ for a large airplane, is capable of supporting an elliptic instability, and even this produces small amplification factors. The instability will be subject to a viscous cut-off. Using the analysis ofLandman \& Saffrnan (1987) one estimates that for a large airplane the cut-off occurs for scales $<14 \mathrm{~cm}$. Down in scale from the cut-off there may be smaller scales due to the non-linear cascade.

\subsection{Dielectric constant variation due to atmospheric stratification}

In the literature it has been postulated that the scattering mechanism for aircraft vortices is "turbulent mixing of atmospheric index of refraction gradients" (Gilson 1994), the turbulence being assumed to be similar to atmospheric turbulence. This sub-section tries to develop a concrete picture for the quoted phrase.

\subsubsection{The 2D picture without velocity fluctuations}

Suppose that the vortex pair descends through a distance $\Delta z=z_{f}-z$ from its altitude of formation, $z_{f}$. As it descends it carries, within an oval, ambient atmospheric fluid from $z_{f}$. The oval has semi-axes $1.045 b_{0}$ and $0.865 b_{0}$ (Milne-Thomson 1968, p. 360) which we shall approximate in the scattering analysis by a cylinder of circular cross-section of radius $a=0.95 b_{0}$.

Let tildes denote fluid properties within the oval and let overbars denote properties in the ambient atmosphere. Then introducing the total pressure, $p=p_{a}+p_{v}$, into (2.1), converting to SI units and eliminating pressure in favor of density using the ideal gas law we get the following equation for the difference in index of refraction between the oval and the surrounding atmosphere:

$$
[\widetilde{n}(z)-\bar{n}(z)] \times 10^{6}=223[\widetilde{\rho}(z)-\bar{\rho}(z)]-36.7\left[\widetilde{\rho}_{v}(z)-\bar{\rho}_{v}(z)\right]+1.08 \times 10^{6}\left[\frac{\tilde{\rho}_{v}(z)}{\widetilde{T}(z)}-\frac{\bar{\rho}_{v}(z)}{\bar{T}(z)}\right]
$$

As it descends, the fluid in the oval compresses adiabatically in response to increasing ambient pressure. A density difference, $\tilde{\rho}(z)-\bar{\rho}(z)$, from the ambient value will remain only if the ambient atmospheric density gradient is different from the adiabatic one. This difference is usually expressed in terms of the parameter $N$, the Brünt Väisälä frequency defined so that for small $\Delta z$ (Pedlosky 1979)

$$
\tilde{\rho}(z)-\bar{\rho}(z)=-\frac{\bar{\rho}(z) N^{2}}{g} \Delta z
$$

The first term in (2.10), which represents the dry air contribution, is thus determined.

Since the pressure of the descending fluid is equalized to the ambient we can obtain its temperature using Eq. (2.11):

$$
\widetilde{T}-\bar{T}(z)=+\frac{\bar{T}(z) N^{2}}{g} \Delta z .
$$

To obtain $\bar{\rho}_{v}(z)$, the ambient vapor density, we introduce the ambient relative humidity $\overline{R H}(z)$ as an input parameter so that $\bar{\rho}_{v}(z)$ can be obtained from

$$
\frac{\bar{\rho}_{v}(z)}{\bar{\rho}(z)}=\frac{\bar{P}_{v}(z)}{\bar{P}(z)}=\frac{\overline{R H}(z) p_{s a t}(\bar{T})}{\bar{P}(z)},
$$

where the function $p_{s a t}(T)$ is given in Reid et al.(1987, p. 657). Finally, to obtain $\tilde{\rho}_{v}(z)$, 


$\begin{array}{lllll}\text { Case } & \text { R.H } & \overline{R H}(z) & N \text { in } s^{-1} \mathrm{~J} \varepsilon_{\mathrm{r}} \\ 1 & 0 & 0 & 0 & 0 \\ 2 & 0 & 0 & .017 & -9.8 \times 10^{-7} \\ 3 & .60 & .60 & 0 & -2.8 \times 10^{-7} \\ 4 & .60 & .60 & .017 & -1.1 \times 10^{-6} \\ 5 & .592 & .603 & 0 & -4.4 \times 10^{-6} \\ 6 & .592 & .603 & .017 & -2.8 \times 10^{-6}\end{array}$

TABLE 1. Dielectric constant in developed in the oval for various atmospheric conditions after $1.5 \tau$ units.

use the fact that all constituents of the descending air compress by the same factor so that:

$$
\frac{\bar{\rho}_{v}\left(z_{f}\right)}{\bar{\rho}\left(z_{f}\right)}=\frac{\tilde{\rho}_{v}(z)}{\tilde{\rho}(z)}
$$

Then introducing the relative humidity at formation $\left(\mathrm{RH}_{f}\right)$ as an input parameter. one obtains:

$$
\tilde{\rho}_{v}(z)=\frac{\mathrm{RH}_{f} p_{\text {sat }}\left(\bar{T}_{f}\right) \bar{\rho}(z)}{\bar{P}(z)}\left[1+\left(\frac{\bar{\rho}(z)}{\bar{P}(z)} g-\frac{N^{2}}{g}\right) \Delta z\right]
$$

The value of $N$ near sea level varies from average summer levels of $0.014 \mathrm{~s}^{-1}$ to mild winter values of $0.02 \mathrm{~s}^{-1}$ to a value of $0.03 \mathrm{~s}^{-1}$ for a Fairbanks winter (W.D. Kriese, Boeing Aircraft, Private Communication; Pedlosky 1979, p. 331). Thus, winter weather produces greater reflectivity than tropical weather (for the same vortex descent altitude and neglecting humidity). What is the range of $\Delta z$ that is relevant for the wake hazard problem? The lifetime of trailing vortices has been measured to range between 1.5 and $8 \tau$ units (Spalart 1998, his fig. 1) where one $\tau$ unit corresponds to the time takes for a vortex pair to descend one vortex separation distance. Hence the minimum separation in time between aircraft that could ever be contemplated (in the absence of techniques to destroy vortices) is $1.5 \tau$ units. A vortex separation distance of $b_{0}=47.88 \mathrm{~m}$ implies that the smallest detectable vor tex descent should be $72 \mathrm{~m}$. This assumes that stratification neither impedes nor speeds the descent.

Table 2.3.1 shows $\Delta \varepsilon_{\mathrm{r}} \approx 2 \Delta n$ for various atmospheric conditions taking $\Delta z=61 \mathrm{~m}$, a slightly more conservative value. We used $\bar{P}(z), \bar{\rho}(z)$, and $\bar{T}(z)$ values for the Standard Atmosphere at sea level. Cise 1 is the trivial case (neutral and dry armosphere). Case 2 is for stably stratified dry arr. Case 3 has a neutral atmosphere with uniform humidity. Case 4 combines the stratification of case 2 with the humidity of case 3 . We see that the total effect is not quite additive. Case 5 uses the vapor pressure for the model atmosphere given in the Handbook of Geophysics (1960, p. 13-5) in which the air is slightly drier at the higher altitude. The resulting $\Delta \varepsilon_{\mathrm{r}}$ is higher than for uniform humidity. Case 6 has both stratification and humidity gradient.

\subsubsection{Dielectric constunt of the oval in Gilson's experiment}

To secure a value for the dielectric constant $\Delta \varepsilon_{\mathrm{r}}$ for the experiment, performed on $K$ wajalein atoll, we used the density and index of refraction vs. height sounding given by Gilson. Since gradients relative to adiabatic are needed, one quantity had to be assumed to obtain them. This was the temperature at flight altitude $(5000 \mathrm{ft})$ which we took to be $295 \mathrm{~K}$ assuming a balmy sea level temperature of $85^{\circ} \mathrm{F}$ and a lapse rate of $-.005 \mathrm{~K} / \mathrm{m}$. By comparing the measured index of refraction with the value for dry air at the local 
density we gathered that $7 \%$ relative humidity must have been present at Hight altitude. The value of the Brünt-Väisälä frequency $N$ was deternined to be $.017 \mathrm{~s}^{-1}$ by comparing the actual density gradient with the adiabatic one. We need the descent altitude of the oval at the first measurement instant 66 secs after aircraft passage. Using the flight speed and nominal aircraft weight provided together with the theory of an elliptically loaded wing, the vortex circulation and vortex separation were inferred to be $\Gamma=387.6 \mathrm{~m}^{2} / \mathrm{s}$ and $b_{0}=53.4 \mathrm{~m}$. For vortex pair descent unaffected by stratification, this yields a descent of $77 \mathrm{~m}$ in 66 secs. The parameter determining the effect of stratification on vortex pair descent is $N_{0}^{*}=2 \pi N b_{0}^{2} / \Gamma$ (Spalart 1996). Its value is 0.79 and using the simulation results of Spalart (1996, his figs. 4 and $10 a$ ) we concluded that that stratification would shorten descent from $77 \mathrm{~m}$ to about $64.6 \mathrm{~m}$. Thus we obtained $\Delta \varepsilon_{\mathrm{r}}=-8.4 \times 10^{-7}$.

\subsubsection{The boundary of the oval}

(1) Laminar boundary Thermal diffusion smooths the gradient in $\Delta \rho$ at the boundary of the oval and the flow sweeps away and replenishes the diffused $\Delta \rho$. This may be locally modelled as a strained diffusion layer: at equilibrium we have (e.g. Leonard et al.1987):

$$
\Delta \rho(\eta)=\Delta \rho(1) \operatorname{erf}\left(\eta / \delta_{\mathrm{diff}}\right), \quad \delta_{\mathrm{diff}} \equiv(2 \kappa / e)^{1 / 2}
$$

where $\eta$ is normal to the interface, $\kappa$ is the thermal diffusivity and $e$ is the strain rate. For a Prandtl number of 0.7 and

$$
e=\frac{\Gamma}{2 \pi\left(b_{0} / 2\right)^{2}}
$$

which represents the strain rate at a distance of half the vortex spacing, one gets

$$
\delta_{\text {diff }}=2.1 b_{0}\left(\frac{\Gamma}{\nu}\right)^{-1 / 2}
$$

which equals $2.2 \mathrm{~cm}$ for Gilson's experiment.

When flaps are deflected (as during a landing) multiple vortices are created on each side of the wake and their interactions can lead to periodic ejection of fluid from the oval and engulfment of ambient fluid. This leads to a stretching and folding of diffusion layer as shown in the simulations of Spalart (1996). However the minimum thickness of the folded layers cannot be smaller than the estimate (2.18).

Any vorticity fluctuations within the core will induce potential flow fluctuations in the outer flow. This will also cause the boundary of the oval to become distorted. Maxworthy (1970) provides experimental evidence for this in the context of vortex rings and RomKedar et al.(1990) provide a geometric description.

(ii) Turbulent boundary In the presence of stratification baroclinic torque generates a shear layer at the boundary of the oval (Spalart 1996). Can this shear layer become turbulent? To answer this, the Reynolds number $R e_{\delta}=(\Delta U / 2) \delta / \nu$ was estimated and compared with a critical Reynolds number. Here $\Delta U$ and $\delta$ are the velocity jump and laminar thickness respectively of the shear layer and were estimated as follows: (i) Spalart (1996) provides a model (his Eq. 6), calibrated using simulations, for the circulation generated in the shear layer. This allows one to infer the average circulation per unit length (hence the average velocity jump $\Delta U$ ) along the oval perimeter. (ii) The shear layer thickness, set by balance between viscous diffusion and vortex induced strain, is given by $\delta \approx \pi^{1 / 2} b_{0} / \sqrt{\Gamma / \nu}$.

The Reynolds number was found to grow linearly with descent height and even with a descent a quarter of what it is at 66 secs, we have $R e_{\delta}=73$ for Gilson's (1992) experiment. According to the analysis of Betchov \& Szewczyk (1963) for a shear layer, this is quite 
unstable with an $e$-folding time of about 2 secs. $\Lambda$ this rate, transition to turbulence in the shear layer would take about 20 secs. In reality, the shear layer is curved. However, since the baroclinic circulation is opposite that of the primary vortices, the circulation decreases outward and so curvature can only further destabilize the layer. Thus we are convinced that the boundary of the oval is turbulent in Gilson's measurements.

The circulation in the Kelvin-Helmholtz vortices can be estimated as the circulation in one wavelength of the instability. From it the strain rate in the braid region can be obtained and thence the thickness of the braids. At $66 \mathrm{secs}$, $\delta_{\text {braids }}=0.45 \mathrm{~cm}$. This is smaller than $\delta_{\text {diff }}$ we expect it to determine the the wavelength at which the RCS cuts off.

\subsubsection{Evidence for the role of stratification in radar reflectivity}

Here we discuss facts immediately accessible from the experiments. Later in the paper we will perform a more quantitative comparison.

(i) Gilson observed increasing RCS with distance behind the plane. Nespor et al.(1994, p. 658) noted that the cross-section tended to increase as the vortex dropped into the lower beams. Their respective interpretations were developing turbulence and growth of the turbulent region (so that a greater fraction of the radar pulse volume consisted of turbulence). However, the observations are more simply explained by the increasing refractive index contrast between the oval and the ambient. Indeed $R C S \propto\left(\Delta \varepsilon_{\mathrm{r}}\right)^{2} \propto$ $(\Delta z)^{2}$ and $(\Delta z) \propto t$ (when the descent is not impeded by stratification). Thus, the radar cross-section should increase by a factor of $4(6 \mathrm{db})$ for every doubling of the distance behind the plane. Several plots in Gilson (1992) show an increase that are a little smaller than this value probably because the descent is impeded by stratification.

(ii) Gilson notes that the RCS decreases with altitude. This is consistent with the decrease in the Brünt- $V$ äisälä frequency, $N$, with altitude.

In conclusion, there is experimental support that atmospheric stratification is playing a role in experiments in the simple way we have described. Since stratification has seasonal, diurnal and geographic variations its use as a basis for detection would have to be combined with atmospheric measurements and would be unusable in neutral conditions or when the vortex descent is strongly impeded by stratification.

\section{Scattering Analysis with the Born Approximation}

\subsection{General formulation}

The scattering analysis for an aircraft wake has two peculiar features that ought to be recognized. (1) The wake is a long target which always fills the incident beam in one direction and is quite coherent along this direction. Thus one cannot follow the type of analysis performed for a collection of rain drops, for instance, where it is assumed that at each point within the beam, a plane wave of uniform intensity and polarization is incident on each drop and the scattered powers of all the drops simply add because the scattered fields are uncorrelated. In the present situation, one has to account for the variation in beam properties across the whole target. (2) More troublesome is the fact that the usual far-field assumption cannot be made because as the distance from the radar to the target is made larger, the illuminated size of the target also increases! More concretely, the condition that should be satisfied in the far or Fraunhoffer zone is:

$$
\epsilon_{\mathrm{HF}}=\frac{\pi L_{\mathrm{s}}^{2}}{\lambda R_{r}} \ll 1,
$$


where $R_{r}$ is the distance from the receiver to the target, $L_{s}$ is a characteristic size of the scattering region and $\lambda$ is the wavelength. The difficulty in satisfying (3.1) for a beam filling target arises from the fact that $L_{s}=R_{t} \Delta \theta / 2$ grows with range $R_{t}$ from the transmitter to the target. Here $\Delta \theta$ is the half-power beamwidth. In particular, for co-located transmitter and receiver (3.1) becomes:

$$
\epsilon_{\mathrm{ff}}=\frac{\pi R_{r}(\Delta \theta)^{2}}{\lambda} \ll 1
$$

Ironically, the far-field condition becomes more difficult to satisfy the further one is from the target. The experiments of Chadwick et al.(1984) and Gilson (1992) we have $6 \leqslant \epsilon_{\mathrm{ff}} \leqslant 15$. Tatarski's (1961) analysis makes the assumptions of a far-field and a uniform incident field. Hence it is not formally valid for a coherent wake. Fortunately, the problem remains tractable even if these assumptions are relaxed.

We consider time harmonic electric and magnetic fields

$$
\overline{\mathbf{E}}(\mathbf{x}, t)=\operatorname{Re}\left[\mathbf{E}(\mathbf{x}) \mathrm{e}^{-\mathrm{i} \omega t}\right], \quad \overline{\mathbf{H}}(\mathbf{x}, t)=\operatorname{Re}\left[\mathbf{H}(\mathbf{x}) \mathrm{e}^{-\mathrm{i} \omega t}\right],
$$

where $\mathbf{E}$ and $\mathbf{H}$ are complex. Rationalized MKS units (Jackson 1962, p. 611) will be used to write the electrodynamic equations. The starting point is the solution to the scattering problem as an integral equation (Ishimaru 1978, vol. 1, p. 16):

$$
\begin{aligned}
\boldsymbol{\Pi}_{\mathrm{s}}(\mathbf{x}) & =\int_{V} \Delta \varepsilon_{\mathbf{r}}\left(\mathbf{x}^{\prime}\right) \mathbf{E}\left(\mathbf{x}^{\prime}\right) G\left(\mathbf{x}, \mathbf{x}^{\prime}\right) d \mathbf{x}^{\prime}, \\
\Delta \varepsilon_{\mathrm{r}}\left(\mathbf{x}^{\prime}\right) & \equiv \frac{\varepsilon\left(\mathbf{x}^{\prime}\right)}{\varepsilon_{0}}-1 .
\end{aligned}
$$

Here

$$
G\left(\mathbf{x}, \mathbf{x}^{\prime}\right)=\frac{\mathrm{e}^{\mathrm{i} k \Delta}}{4 \pi \Delta}, \quad \Delta \equiv\left|\mathbf{x}-\mathbf{x}^{\prime}\right|
$$

is the free space Green's function and $\Pi_{s}$ is the Hertz vector of the scattered field:

$$
\mathbf{E}_{\mathrm{s}}=\nabla \times \nabla \times \boldsymbol{\Pi}_{\mathrm{s}}, \quad \mathbf{H}_{\mathrm{s}}=-\mathrm{i} \omega \varepsilon_{0} \nabla \times \boldsymbol{\Pi}_{\mathbf{s}} .
$$

To calculate $\mathbf{E}$ and $\mathbf{H}$ from $\boldsymbol{\Pi}$ requires evaluating curls (with respect to $\mathbf{x}$ ) of the integrand in (3.4). We list them here for completeness. Denoting by $\mathbf{C}=\Delta \varepsilon_{\mathbf{r}}\left(\mathbf{x}^{\prime}\right) \mathbf{E}\left(\mathbf{x}^{\prime}\right)$ the vector in the integrand which is constant with respect to $\mathbf{x}$ we get:

$$
\begin{aligned}
\nabla \times \mathbf{C} G & =\nabla G \times \mathbf{C}, \\
\nabla \times \nabla \times \mathbf{C} G & =\nabla \times(\nabla G \times \mathbf{C})=(\mathbf{C} \cdot \nabla) \nabla G-\mathbf{C} \nabla^{2} G .
\end{aligned}
$$

Equations (3.8) and (3.9) involve first and second derivatives of $G$ which are:

$$
\begin{aligned}
\frac{\partial G}{\partial x_{i}} & =\frac{1}{4 \pi} \frac{\mathrm{e}^{\mathrm{i} k \Delta}}{\Delta^{2}} x_{i}(i k-1 / \Delta), \\
\frac{\partial^{2} G}{\partial x_{i} \partial x_{j}} & =\frac{1}{4 \pi} \frac{\mathrm{e}^{\mathrm{i} k \Delta}}{\Delta^{4}}\left[\left(\Delta^{2} \delta_{i j}+\mathrm{i} k x_{i} x_{j} \Delta-2 x_{i} x_{j}\right)(\mathrm{i} k-1 / \Delta)+x_{i} x_{j} / \Delta\right],
\end{aligned}
$$

where $\delta_{i j}$ is the Kronecker delta.

\subsection{The Born approximation}

The integrand in (3.4) contains the total electric field (incident + scattered) which is in general unknown, however, since in the present case $\Delta \varepsilon_{\mathrm{r}}=\mathcal{O}\left(10^{-6}\right)$ one can invoke the Born approximation that it can be set equal to the incident field. This corresponds to retaining only the first term in a Neumann series solution to the integral equation. The 


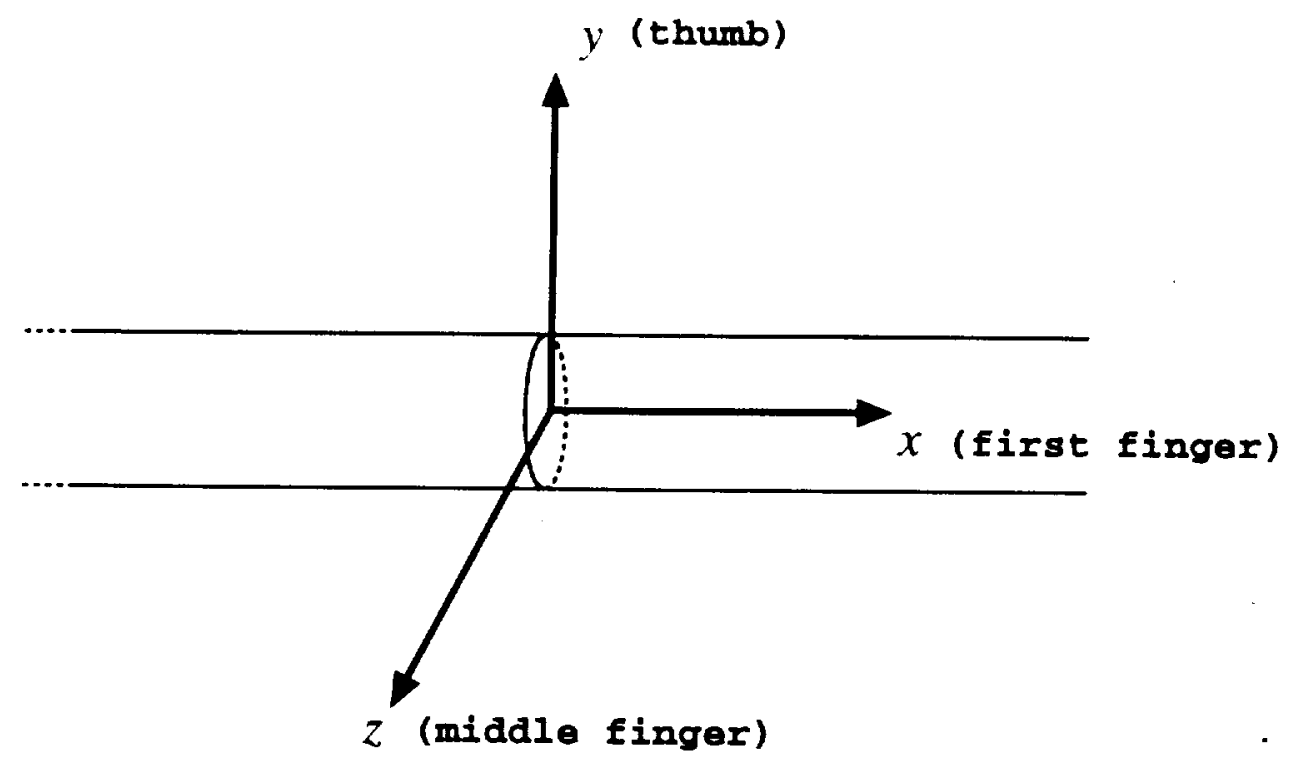

FiguRE 3. Wake coordinate system. Use the indicated fingers of the left hand to follow the transformations (3.15) $-(3.19)$ discussed in the paper.

Born approximation is valid when the scattered electric field is much smaller than the incident field in the region of the target. Equations (3.4), (3.9) and (3.11) imply that the following three conditions have to be satisfied for this to be true:

$$
\Delta \varepsilon_{\mathrm{r}}\left\{1, k L_{\mathrm{s}},\left(k L_{\mathrm{s}}\right)^{2}\right\} \ll 1
$$

It should be noted that Ishimaru (1978) gives only the second condition perhaps because he was thinking of scattering by particles smaller than a wavelength. In that case $k L_{\mathrm{s}}<1$ and the second condition is the more stringent one.

\subsection{Calculation of the radar cross-section}

The definition of radar cross-section RCS (e.g. Skolnik 1970, p. 1-4) can be written as:

$$
\mathrm{RCS}=4 \pi R_{r}^{2} \frac{\text { power flux density spatially averaged over the receiving aperture }}{\text { spatial maximum of power flux density across incident beam at target }} .
$$

Power flux density has diriensions of power per unit area. The numerator in (3.13) will be evaluated at a single point which is valid if the size of the receiving aperture is smaller than $R_{r}$, an assumption which is difficult to violate. Both numerator and denominator will be evaluated as time averages. The time averaged scattered power flux density vector is

$$
\mathbf{S}_{\mathbf{s}}(\mathbf{x})=\frac{1}{2} \operatorname{Re}\left\{\mathbf{E}_{\mathbf{s}}(\mathbf{x}) \times \mathbf{H}_{\mathbf{s}}{ }^{*}(\mathbf{x})\right\}
$$

where $R e$ denotes the real part. The numerator in (3.13) was evaluated as $\left|\mathbf{S}_{\mathbf{s}}(\mathbf{x})\right|$. As a check, the numerator was also evaluated as $\mathbf{S}_{i}(\mathbf{x}) \cdot \widehat{\mathbf{o}}$ where $\hat{\mathbf{o}}$ is a unit vector (defined in (3.25) below) from the target to the receiver. A difference between the two was never detected.

Appendix A.2 shows that the denominator in $(3.13)$ is simply $(1 / 2) c \varepsilon_{0}$. 


\subsection{Coordinate systems}

We choose the coordinate system of $x$ and $x^{\prime}$ in the integral (3.4) to be located in the wake with a convenient origin. In particular for an axisymmetric target the origin is placed at the target axis as shown in figure 3 , its axial location being chosen to coincide with the axial location where the beam centerline intersects the $x y$ plane. In particular the beam centerline is chosen to intersect the $x y$ plane at $\left(x=0, y=y_{0}\right), y_{0}$ being the bearn pointing error.

Appendix A obtains the electric field in the incident bearn given the electric field on a surface (the aperture) near the antenna. The incident field is given using coordinates centered on the aperture. In the body of the paper we use $\left(x^{*}, y^{*}, z^{*}\right)$ for aperture coordinates. The plane of the aperture is $x^{*} y^{*}$ while $z^{*}$ points normal to the aperture. We now relate beam coordinates $\left(x^{*}, y^{*}, z^{*}\right)$ to wake coordinates $(x, y, z)$ by performing a series of rotations and translations. These transformations serve to define the viewing angles $\left(\alpha_{t}, \beta_{t}\right)$ of the target from the transmitter. Similarly, $\left(\alpha_{r}, \beta_{r}\right)$ are viewing angles from the receiver.

Imagine that the transmitter is looking at the wake from the same side as the reader is looking at figure 3. This figure also shows which fingers of the left hand the reader may employ to visualize the transformations. The reader's palm will eventually become the plane of the aperture, the middle finger will become the direction of propagation, and the first finger will become the direction of polarization. The first transformation turns $z$ (the middle finger) away from the reader so that it can eventually become the beam axis. The $x$-axis (first finger) is also simultaneously flipped. The second transformation shifts the origin to $\left(x=0, y=y_{0}\right)$ to ensure that the beam axis intersects the $x y$ plane where we want it. The coordinates resulting from the first two transformations are therefore

$$
\left(\begin{array}{l}
x^{(1)} \\
y^{(1)} \\
z^{(1)}
\end{array}\right)=\left(\begin{array}{c}
-x \\
y-y_{0} \\
-z
\end{array}\right)
$$

Next, rotate the coordinate system by angle $\beta_{t} \in[0,2 \pi]$ around $x^{(1)}$ axis. This is a counter clockwise rotation about the first finger. Thus $\beta_{t}$ is an elevation angle to the target. We have

$$
\left(\begin{array}{l}
x^{(2)} \\
y^{(2)} \\
z^{(2)}
\end{array}\right)=\left(\begin{array}{ccc}
1 & 0 & 0 \\
0 & \cos \beta_{t} & -\sin \beta_{t} \\
0 & \sin \beta_{t} & \cos \beta_{t}
\end{array}\right)\left(\begin{array}{c}
-x \\
y-y_{0} \\
-z
\end{array}\right) .
$$

Next is a rotation by angle $\alpha_{t} \in[-\pi / 2, \pi / 2]$ about the $y^{(2)}$ axis. This is a counterclockwise rotation about the thumb and we get

$$
\left(\begin{array}{l}
x^{(3)} \\
y^{(3)} \\
z^{(3)}
\end{array}\right)=\left(\begin{array}{ccc}
\cos \alpha_{t} & 0 & -\sin \alpha_{t} \\
0 & 1 & 0 \\
\sin \alpha_{t} & 0 & \cos \alpha_{t}
\end{array}\right)\left(\begin{array}{l}
x^{(2)} \\
y^{(2)} \\
z^{(2)}
\end{array}\right) .
$$

Next, translate the coordinate system in the negative $z^{(3)}$ direction by the range $R_{t}$ from the transmitter to the point $\mathbf{x}=\left(0, y_{0}, 0\right)$ on the target:

$$
\left(\begin{array}{l}
x^{(4)} \\
y^{(4)} \\
z^{(4)}
\end{array}\right)=\left(\begin{array}{c}
x^{(3)} \\
y^{(3)} \\
z^{(3)}
\end{array}\right)+\left(\begin{array}{c}
0 \\
0 \\
R_{t}
\end{array}\right)
$$

Finally, to the get the polarization vector oriented correctly, the coordinate system is rotated by angle $\gamma$ about the $z^{(4)}$ axis (about the middle finger, counter-clockwise looking 
into the palm):

$$
\left(\begin{array}{l}
x^{*} \\
y^{*} \\
z^{*}
\end{array}\right)=\left(\begin{array}{ccc}
\cos \gamma & \sin \gamma & 0 \\
-\sin \gamma & \cos \gamma & 0 \\
0 & 0 & 1
\end{array}\right)\left(\begin{array}{l}
x^{(4)} \\
y^{(4)} \\
z^{(4)}
\end{array}\right) .
$$

The $x^{*}$ axis (first finger) now points along the polarization direction. Putting all the transformations together gives:

$$
\begin{aligned}
x^{*}= & \cos \gamma\left\{-x \cos \alpha_{t}-\sin \alpha_{t}\left[\left(y-y_{0}\right) \sin \beta_{t}-z \cos \beta_{t}\right]\right\}+ \\
& \sin \gamma\left\{\left(y-y_{0}\right) \cos \beta_{t}+z \sin \beta_{t}\right\}, \\
y^{*}= & \cos \gamma\left\{\left(y-y_{0}\right) \cos \beta_{t}+z \sin \beta_{t}\right\}- \\
& \sin \gamma\left\{-x \cos \alpha_{t}-\sin \alpha_{t}\left[-z \cos \beta_{t}+\left(y-y_{0}\right) \sin \beta_{t}\right]\right\}, \\
z^{*}= & R_{t}-x \sin \alpha_{t}+\cos \alpha_{t}\left[-z \cos \beta_{t}+\left(y-y_{0}\right) \sin \beta_{t}\right] .
\end{aligned}
$$

The incident electric field is in the $\widehat{\mathbf{x}}^{*}$ direction and to obtain its $(x, y, z)$ components for use in the integral (3.4) we note that

$$
\begin{aligned}
\widehat{\mathbf{x}}^{*} & =-\cos \alpha_{t} \cos \gamma \widehat{\mathbf{x}}+\left(\cos \beta_{t} \sin \gamma-\cos \gamma \sin \alpha_{t} \sin \beta_{t}\right) \hat{\mathbf{y}}+ \\
& \left(\sin \beta_{t} \sin \gamma+\cos \gamma \sin \alpha_{t} \cos \beta_{t}\right) \widehat{\mathbf{z}}
\end{aligned}
$$

from (3.20). Just as the "incident direction" $\hat{\mathrm{i}}$ from the transmitter to the point $\left(0, y_{0}, 0\right)$ is

$$
\widehat{\mathbf{i}}=\widehat{\mathbf{z}}^{*}=-\sin \alpha_{t} \widehat{\mathbf{x}}+\cos \alpha_{t} \sin \beta_{t} \widehat{\mathbf{y}}-\cos \alpha_{t} \cos \beta_{t} \widehat{\mathbf{z}},
$$

we define the "observer direction" $\widehat{o}$ from the point $\left(0, y_{0}, 0\right)$ to the receiver in a similar way but using angles $\alpha_{r}$ and $\beta_{r}$ and reversing the sign of the vector:

$$
\widehat{\mathbf{o}}=\sin \alpha_{r} \widehat{\mathbf{x}}-\cos \alpha_{r} \sin \beta_{r} \widehat{\mathbf{y}}+\cos \alpha_{r} \cos \beta_{r} \widehat{\mathbf{z}},
$$

The location of the receiver is then:

$$
\mathbf{x}_{r}=y_{0} \widehat{\mathbf{y}}+R_{r} \widehat{\mathbf{o}} .
$$

\subsection{Numerical integration procedure}

The numerical integrations required to evaluate the integral (3.4) were performed using two methods.

Method A: This is the more general but less efficient of the two methods. It uses Simpson's rule in cylindrical coordinates $(x, r, \phi)$. The integral over the infinite direction $x$ was truncated at $|x|=3 u_{\mathrm{b}}$ where $u_{\mathrm{b}}=R_{t} \sin \theta_{\mathrm{b}}$ is the characteristic beam radius at the target, $\theta_{\mathrm{b}}$ being the half beamwidth. In the radial direction, the integrals were iruncated at $\tau=3 \delta_{\varepsilon}$ when the vortex was the target. When the oval is the target, the integrand is zero for $r>a$. The number of quadrature points in each direction was determined from the input parameter $N_{q l}$ which is the number of quadrature intervals desired per characteristic length $l$ of fluctuations of the integrand. The characteristic length in each direction was chosen as follows:

$$
l_{x}=\min \left(\lambda, u_{\mathrm{b}}\right), \quad l_{\phi}=\min \left(\lambda, u_{\mathrm{b}}\right), \quad l_{r}=\min \left(\lambda, u_{\mathrm{b}}, \delta_{r}\right)
$$

where

$$
\delta_{r}= \begin{cases}\delta_{\varepsilon}, & \text { if the vortex is the target, } \\ (1 / 2) a, & \text { if the oval is the target. }\end{cases}
$$

If the length of an integration is $L$ then $N_{l}=\operatorname{int}(L / l)+1$ characteristic lengths cover the integration length and the number of quadrature intervals was determined as $N_{l}=$ 
$N_{l l}$ max $\left(3, N_{l}\right)$, the purpose of the max being to ensure a minimum number of cuadrature points regardless. For each calculation of radar cross-section presented for this method we began with $N_{\eta l}=6$ and kept on doubling it until two successive doublings showed no perceptible change in the radar cross-section. Computational cost prevented use of this method above $f \approx 0.16 \mathrm{GHz}$.

Method B: This method was developed to efficiently calculate the radar cross-section for the oval at the relatively high frequencies of Gilson's (1992) experiment. The implementation is currently specialized to backscattering for the oval (and for the oval with a slice of ambient fluid; see figure 8). For brevity we will describe the method for normal incidence and $\beta_{t}=0$.

Each of the six integrals needed to evaluate the scattered $\mathbf{E}$ and $\mathbf{H}$ fields is of the form:

$$
I=\int A(x, y, z) \mathrm{e}^{\mathrm{i} \boldsymbol{\phi}(\boldsymbol{x}, y, z)} d x d y d z,
$$

where $z$ is the direction along the beam axis and the cross-beam coordinates are $(x, y)$. The basic idea of the method is to divide the integration interval in a given direction (say $\zeta$ ) into many sub-intervals and to approximate the amplitude and phase in each sub-interval as a linear function of $\zeta$ :

$$
I=\int_{\zeta_{1}}^{\zeta_{2}} A(\zeta) \mathrm{e}^{\mathrm{i} \phi(\zeta)} d \zeta \approx \Delta \zeta \int_{0}^{1}\left(A\left(\zeta_{1}\right)+\xi \Delta A\right) \mathrm{e}^{\mathrm{i}\left(\phi\left(\zeta_{1}\right)+\xi \Delta \phi\right)} d \xi
$$

where

$$
\Delta A \equiv A\left(\zeta_{2}\right)-A\left(\zeta_{1}\right), \quad \Delta \phi \equiv \phi\left(\zeta_{2}\right)-\phi\left(\zeta_{1}\right) .
$$

The integral in each sub-interval can then be obtained analytically:

$$
I \approx-\frac{\mathrm{i}}{\Delta \phi} \Delta \zeta\left\{-\left[A\left(\zeta_{1}\right)+\frac{\mathrm{i}}{\Delta \phi} \Delta A\right] \mathrm{e}^{\mathrm{i} \phi\left(\zeta_{1}\right)}+\left[A\left(\zeta_{2}\right)+\frac{\mathrm{i}}{\Delta \phi} \Delta A\right] \mathrm{e}^{\mathrm{i} \phi\left(\zeta_{2}\right)}\right\}
$$

The integral along the beam ( $z$ direction) was done first and tests revealed that one sub-interval was sufficient to obtain an accurate integral for each region. For the sliced oval, one interval for each piece was used. The resulting integral over an interval has the form (3.32) with $\zeta$ replaced by $z$. Now the two terms in the result (3.32), when considered as a function of the remaining coordinates, are again in the basic form (3.30) with amplitudes and phases a function of $x$ and $y$. Hence, the same method can be applied successively in these directions. In these directions hundreds of sub-intervals were required for convergence.

\section{Approximate scattering analysis}

\subsection{General formulation}

To obtain analytical insight into the numerical results and as a check on the numerics for special cases, it is useful to have closed form expressions which approximate the radar cross-section. Such expressions are developed in this section and, later in the paper, their predictions will be compared with the formulation of the previous section.

Four assumptions will be invoked. The first assumption is that of the far-field which holds when (3.1) is satisfied. In this case the scattered electric field implied by (3.4) is given by (see Ishimaru 1978, vol. 1, p. 17):

$$
\begin{aligned}
\mathbf{E}_{\mathbf{s}}(\mathbf{x}) & =\mathbf{f}(\widehat{\mathbf{o}}, \hat{\mathbf{i}}) \mathrm{e}^{\mathrm{i} k R_{r}} / R_{r} \\
\mathbf{f}(\widehat{\mathbf{o}}, \hat{\mathbf{i}}) & \equiv \frac{k^{2}}{4 \pi} \int_{V}\left\{-\hat{\mathbf{o}} \times\left[\widehat{\mathbf{o}} \times \mathbf{E}\left(\mathbf{x}^{\prime}\right)\right]\right\} \Delta \varepsilon_{\mathrm{r}}\left(\mathbf{x}^{\prime}\right) \mathrm{e}^{-\mathrm{i} k \mathbf{x}^{\prime} \cdot \hat{\mathbf{o}}} d \mathbf{x}^{\prime},
\end{aligned}
$$


The second assumption is the Born approximation of the previous section: $\mathbf{E}\left(\mathbf{x}^{\prime}\right) \longrightarrow$ $\mathbf{E}_{\mathbf{i}}\left(\mathbf{x}^{\prime}\right)$ in the integrand.

The third approximation replaces the conical incident beam by a cylindrical beam in the vicinity of the target as explained in Appendix A.2. The incident electric field then has the form

$$
\mathbf{E}_{\mathrm{i}}(\mathbf{x})=\hat{\mathbf{x}}^{*} F(u ; \sigma) \mathrm{e}^{\mathrm{i} k z^{*}},
$$

where

$$
u \equiv\left(x^{* 2}+y^{* 2}\right)^{1 / 2}
$$

is the normal distance from the beam axis. Substituting for $z^{*}$ from (3.20) and using (3.24), the incident electric field takes the form

$$
\mathbf{E}_{\mathbf{i}}(\mathbf{x})=\widehat{\mathbf{x}}^{*} F(u ; \sigma) e^{i k \mathbf{x} \cdot \hat{\mathbf{i}}} \mathrm{e}^{i k \Phi}
$$

where

$$
\Phi \equiv R_{t}-y_{0} \cos \alpha_{t} \sin \beta_{t}
$$

Two types of beam shapes, justified in the appendix, will be considered. The first type is Gaussian:

$$
F(u ; \sigma)=\mathrm{e}^{-u^{2} / \sigma^{2}}
$$

and corresponds to a Gaussian illumination of the transmitting aperture. The second type corresponds to that produced by a uniformly illuminated circular aperture:

$$
F(u ; \sigma)=\frac{2 J_{1}(u / \sigma)}{(u / \sigma)}
$$

Using (3.20) we have

$$
u^{2}=x^{* 2}+y^{* 2}=A x^{2}+B x\left(y-y_{0}\right)+C x z+D\left(y-y_{0}\right)^{2}+E\left(y-y_{0}\right) z+F z^{2}
$$

in which

$$
\begin{array}{lll}
A=\cos ^{2} \alpha_{t}, & B=\sin 2 \alpha_{t} \sin \beta_{t}, & C=-\cos \beta_{t} \sin 2 \alpha_{t} \\
D=\cos ^{2} \beta_{t}+\sin ^{2} \alpha_{t} \sin ^{2} \beta_{t}, & E=\cos ^{2} \alpha_{t} \sin 2 \beta_{t}, & F=\cos ^{2} \beta_{t} \sin ^{2} \alpha_{t}+\sin ^{2} \beta_{t}
\end{array}
$$

Keeping all the terms in (4.9) makes the resulting integrals analytically intractable. Thus we make the fourth approximation that the beam is much wider than the cross-sectional dimensions of the wake. In the wide beam limit, the beam amplitude varies only along the axis of the wake an: is constant within each cross-section of the wake. Thus setting $y=z=0$ in (4.9) we $g_{i t}$.

$$
u^{2}=x^{* 2}+y^{* 2}=A x^{2}-B x y_{0}+D y_{0}^{2}
$$

Substituting (4.5) into (4.2) gives:

$$
\mathbf{f}(\widehat{\mathbf{o}}, \hat{\mathbf{i}})=\frac{\mathbf{s} k^{2} \mathrm{e}^{\mathrm{i} k \phi}}{4 \pi} \int_{V} \Delta \varepsilon_{\mathbf{r}}\left(\mathbf{x}^{\prime}\right) F(u ; \sigma) \mathrm{e}^{\mathrm{i} k \mathbf{x}^{\prime} \cdot(\hat{\mathbf{i}}-\hat{\mathbf{o}})} d \mathbf{x}^{\prime},
$$

where

$$
\mathbf{s} \equiv-\hat{\mathbf{o}} \times\left[\hat{\mathbf{o}} \times \hat{\mathbf{x}}^{*}\right]
$$

We have $|\mathbf{s}|=\sin \chi$ where $\chi$ is the angle between $\widehat{\mathbf{x}}^{*}$ and $\widehat{\mathbf{o}}$.

To calculate the scattered power flux we need the scattered magnetic field correspond- 
ing to (4.1). Using the Maxwell equation

$$
\mathbf{H}_{\mathrm{s}}^{*}=\frac{1}{\mathrm{i} \omega \mu_{0}} \nabla \times \mathbf{E}_{\mathrm{s}}
$$

one obtains

$$
\mathbf{H}_{\mathrm{s}}{ }^{*}=\frac{k}{R_{r} \omega \mu_{0}} \mathrm{e}^{-\mathrm{i} k R_{r}}(\hat{\mathbf{o}} \times \mathbf{f})^{*} .
$$

Substituting (4.15) into (3.14) and noting that according to (4.12) $\mathbf{f}$ is of the form $\mathbf{f}=\mathbf{s}$ where $\mathbf{s}$ is a real vector perpendicular to $\widehat{o}$ and $\mathcal{C}$ is a complex number, we conclude that

$$
\mathbf{S}_{\mathrm{s}}(\mathbf{x})=\frac{k}{2 R_{r}^{2} \omega \mu_{0}}|\mathbf{f}|^{2} \widehat{\mathbf{o}} .
$$

The incident flux in the definition of the radar cross-section (3.13) is taken to be that for the original conical beam, namely, $c \varepsilon_{0} / 2$. Hence (3.13) gives simply:

$$
\mathrm{RCS}=4 \pi|\mathbf{f}|^{2} .
$$

Finally, let us keep ready at hand the following geometric quantity which appears in Eq. (4.12):

where

$$
\mathbf{x} \cdot(\widehat{\mathbf{i}}-\widehat{\mathbf{o}})=-P x-Q y-S z
$$

$$
\begin{aligned}
& P \equiv \sin \alpha_{t}+\sin \alpha_{r} \\
& Q \equiv-\cos \alpha_{t} \sin \beta_{t}-\cos \alpha_{r} \sin \beta_{r} \\
& S \equiv \cos \alpha_{t} \cos \beta_{t}+\cos \alpha_{r} \cos \beta_{r}
\end{aligned}
$$

\subsection{Scattering off a Fourier mode}

Begin by Fourier decomposing the dielectric constant in the axial and azimuthal directions:

$$
\Delta \varepsilon_{\mathrm{r}}(\mathbf{x})=\sum_{k_{z}, m} \Delta \widehat{\varepsilon}_{\mathrm{r}}\left(r ; k_{x}, m\right) \mathrm{e}^{i\left(k_{z} x+m \phi\right)} .
$$

To justify the sum in $k_{x}$ instead of an integral, pretend that there is a periodicity length $L_{x}$ and then let $L_{x} \rightarrow \infty$.

Substituting the Fourier expansion (4.22) and the Gaussian incident beam (4.7) into (4.12) gives:

$$
\mathbf{f}=\sum_{k_{z}, m} \widehat{\mathbf{f}}\left(k_{x}, m\right)
$$

where

$$
\widehat{\mathbf{f}}\left(k_{x}, m\right)=\frac{s k^{2} \mathrm{e}^{\mathrm{i} k \phi}}{4 \pi} I_{x} \int_{0}^{\infty} r \mathrm{~d} r \Delta \widehat{\varepsilon}_{\mathbf{r}}\left(r ; k_{x}, m\right) I_{\phi}(r),
$$

and $I_{\phi}$ and $I_{x}$ are the following integrals (for a Gaussian beam):

$$
\begin{aligned}
& I_{\phi}=\int_{0}^{2 \pi} \mathrm{d} \phi \mathrm{e}^{-\mathrm{i} k r(S \cos \phi+Q \sin \phi)} \mathrm{e}^{\mathrm{i} m \phi}, \\
& I_{x}=\mathrm{e}^{-D y_{0}^{2} / \sigma^{2}} \int_{-\infty}^{\infty} \mathrm{d} x \mathrm{e}^{-\left(A x^{2}-B x y_{0}\right) / \sigma^{2}} \mathrm{e}^{-\mathrm{i} x\left(k P-k_{*}\right)} .
\end{aligned}
$$

To perform the integral with respect to $\phi$ let $k Q=\tilde{k} \sin \phi_{1}, k S=\tilde{k} \cos \phi_{1}$, so that 
$\tilde{k}=k\left(S^{2}+Q^{2}\right)^{1 / 2}$ and

$$
I_{\phi}=\int_{0}^{2 \pi} d \phi \mathrm{e}^{\mathrm{im} \phi_{1}} \mathrm{e}^{-\mathrm{i} \tilde{k} r \cos \left(\phi-\phi_{1}\right)}
$$

Using an integral tabulated in Gradshteyn \& Ryzhik (1980) (p. 482) we get:

$$
I_{\phi}=2 \mathrm{e}^{\mathrm{i} m \phi_{1}} \mathrm{i}^{m} \pi \cdot J_{m}(-\tilde{k} r)
$$

To evaluate $I_{\ell}$, let $k^{\prime}=k P-k_{x}$ and complete the square on the exponent to get

$$
I_{x}=\frac{\pi^{1 / 2} \sigma}{A^{1 / 2}} \mathrm{e}^{\left(y_{0} / \sigma\right)^{2}\left[B^{2} /(4 A)-D\right]} \mathrm{e}^{-\mathrm{i} k^{\prime} B y_{0} /(2 A)} \mathrm{e}^{-\left(k^{\prime} \sigma\right)^{2} /(4 A)}
$$

Note that there is a Gaussian cut-off factor if the bean is off-center: $\exp \left\{\left[B^{2} /(4.4)-\right.\right.$ $\left.D]\left(y_{0} / \sigma\right)^{2}\right\}$, where the argument can be shown to be negative definite. This corresponds to the "blip" as the radar scans across the vortex. Setting $y_{0}=0$ one obtains

$$
\widehat{\mathbf{f}}\left(k_{x}, m\right)=\frac{s k^{2} \mathrm{e}^{i k R_{t}}}{2} \frac{\pi^{1 / 2} \sigma}{A^{1 / 2}} \mathrm{e}^{-\left(k^{\prime} \sigma\right)^{2} /(4 A)} \mathrm{e}^{\mathrm{i} m \phi_{1}} \mathrm{i}^{m} \int_{0}^{\infty} r d r \Delta \varepsilon_{\mathrm{r}}\left(r ; k_{x}, m\right) J_{m}(-\tilde{k} r)
$$

This has a cut-off due to finite beam size as represented by the factor $\mathrm{e}^{-\left(\boldsymbol{k}^{\prime} \sigma\right)^{2} /(\mathbf{A} A)}$ $\left(k^{\prime} \equiv k P-k_{x}\right)$. It implies that the contribution to the radar cross-section versus frequency by a fluctuation in refractive index at wavenumber $k_{x}$ is a "bump" which is centered at radio wavenumber $k=k_{x} / P$ and which has a half-width of $\delta k=2 A^{1 / 2} /(P \sigma)$. This result is analogous to the Bragg condition of Tatarski (1961). The cut-off is eliminated when it has infinite width, i.e. when $P=\sin \alpha_{r}+\sin \alpha_{t}=0$. This holds if the receiver is at the direction of specular reflection $\left(\alpha_{r}=-\alpha_{t}\right)$. Note that for backscattering $\left(\alpha_{r}=\alpha_{t}\right)$, perfectly normal incidence $\left(\alpha_{r}=\alpha_{t}=0\right)$ is required to eliminate the cut-off. The cut-off is also eliminated if $\alpha_{r}=\alpha_{t} \pm \pi$ and it corresponds to the receiver being on the other side of the transmitter relative to the wake.

For the Bessel function beam the analysis is very similar; only the integral $I_{x}$ is different:

$$
I_{x}=\int_{-\infty}^{\infty} \mathrm{d} x \frac{2 J_{1}(u / \sigma)}{u / \sigma} \mathrm{e}^{-\mathrm{i} k^{\prime} x}=\frac{4 \sigma}{A} \begin{cases}{\left[1-\left(k^{\prime} \sigma\right)^{2} / A\right]^{1 / 2},} & 0 \leqslant k^{\prime} \sigma / A^{1 / 2}<1, \\ 0, & k^{\prime} \sigma / A^{1 / 2} \geqslant 1,\end{cases}
$$

where $u=\left(A x^{2}\right)^{1 / 2}$. In deriving (4.31) the beam offset $y_{0}$ was set, to zero for analytical tractability. Thus one sees that the Gaussian cut-off for the Gaussian beam has been replaced by a sharp cut-off and the same conditions hold for the elimination of this cut-off.

\subsection{Approximate scattering by three mechanisms}

Each of the following three sub-sub-sections specialize the approximate scattering analysis to each of the three mechanisms discussed in $\S 2$.

\subsubsection{Radial density gradient. $2 r_{2}$ the vortex}

We will represent the radial variation $\left(k_{x}=m=0\right)$ of dielectric constant shown in figure 1 by a Gaussian:

Then the radial integral in $(4.30)$ is

$$
\Delta \varepsilon_{\mathrm{r}}(r)=\Delta \varepsilon_{\mathrm{r}}^{0} \exp \left(-r^{2} / \delta_{\epsilon}^{2}\right) .
$$

$$
I_{r}=\Delta \varepsilon_{r}^{0} \int_{0}^{\infty} r \mathrm{~d} r \mathrm{e}^{-r^{2} / \delta_{\varepsilon}^{2}} J_{0}(\tilde{k} r)=\Delta \varepsilon_{r}^{0} \frac{\delta_{\varepsilon}^{2}}{2} \mathrm{e}^{-\tilde{k}^{2} \delta_{c}^{2} / 4}
$$


This has a cut-off due to finite vortex core size which is elinninated when $\widetilde{k}=k\left(S^{2}+\right.$ $\left(Q^{2}\right)^{1 / 2}=0$. This is satisfied for three cases. The first is for incident and scattering directions that are along the vortex. We eliminate this as a possibility since the assumption that the beam strength does not vary in the cross-section of the target becomes invalid. The second case corresponds to $\alpha_{t}=\alpha_{r}$ and $\beta_{r}=\beta_{t}+\pi$. The third case corresponds to forward scattering $\left(\alpha_{r}=-\alpha_{t}, \beta_{r}=\beta_{t}+\pi\right)$. For this case, the cut-off due to beam width is also eliminated and therefore this is the optimal arrangement.

\subsubsection{Three-dimensional fluctuations within the vortex}

As discussed in $\$ 2.2$ three-dimensional fuctuations should arise in the inner part of the vortex cores from the elliptic instability. The azimuthal mode excited by this instability is $m=1$ and for this mode we model index of refraction using known forms for velocity eigenfunctions:

$$
\Delta \hat{\varepsilon}_{\mathrm{r}}\left(r ; k_{x}, m=1\right)=\epsilon \Delta \varepsilon_{\mathrm{r}}^{0} J_{1}\left(k_{r} r\right) \mathrm{e}^{-r^{2} / \delta^{2}}
$$

where $\epsilon$ is the amplitude of the disturbance and $k_{r}$ is the radial wavenumber which we set to $k_{r}=\sqrt{3} k_{x}$. This value corresponds to the unstable wavevectors which lie on a $60^{\circ}$ cone. The resulting radial integral in Eq. (4.30) is given in Gradshteyn \& Ryzhik (1980) (p. 718, 6.633.2):

$$
I_{r}=\frac{1}{2} \epsilon \Delta \varepsilon_{\mathrm{r}}^{0} \delta_{\varepsilon}^{2} \exp \left[-\frac{1}{4}\left(k_{r}^{2}+\widetilde{k}^{2}\right] \delta^{2}\right) I_{1}\left(\frac{k_{r} \widetilde{k} \delta_{\varepsilon}^{2}}{2}\right),
$$

For large arguments the modified Bessel function $I_{1}$ has an exponential factor and therefore the last two factors in (4.35) can be combined to give a Gaussian bump with respect to frequency:

$$
I_{r} \approx \frac{\epsilon \Delta \varepsilon_{\mathrm{r}}^{0} \delta_{\varepsilon}}{\sqrt{\pi k_{r} \tilde{k}}} \exp \left[-\frac{\delta_{\varepsilon}^{2}}{4}\left(k_{r}-\widetilde{k}\right)^{2}\right] .
$$

The center and half width of the bump are given by

$$
k_{c}=\frac{\sqrt{3} k_{x}}{\left(S^{2}+Q^{2}\right)^{1 / 2}}, \quad \Delta k=\frac{2 / \delta_{\varepsilon}}{\left(S^{2}+Q^{2}\right)^{1 / 2}} .
$$

We also have a Gaussian bump in Eq. (4.30) due to finite beam width. In order that the two bumps not annihilate each other they must be very close. In particular the reflectivity is maximized when they occur at the same frequency. The condition for this is:

$$
S^{2}+Q^{2}=3 P^{2}
$$

For backscattering Eq. (4.38) simplifies to $\cot \alpha_{t}= \pm \sqrt{3}$ or $\alpha= \pm 30^{\circ}$. Thus the radar beam must be aligned with the wavevector of the instability. As $P \rightarrow 0$, i.e. as we approach specular reflection the peak of the beamwidth bump moves to higher frequency and its width becomes larger. In order to maintain reflectivity the second bump (Eq. 4.36) must also move out to higher frequency. This is accomplished by letting $\left(S^{2}+Q^{2}\right)^{1 / 2} \rightarrow 0$, i.e. by approaching forward scattering. The increase in frequency yields an increase in radar cross-section due to the leading $k^{2}$ factor in (4.30).

\subsubsection{Oval}

As mentioned in $\$ 2.3 .1$ the oval can be approximated as a cylinder of radius $a$ ( $k_{x}=$ $m=0$ ). In the absence of fluctuations and thermal diffusion the refractive index will be constant inside the cylinder and zero outside. In this case the radial integral in Eq. (4.30) 
is

$$
\Delta \varepsilon_{\mathrm{r}}^{0} \int_{0}^{a} r \mathrm{~d} r \cdot J_{0}(\tilde{k} r)=\frac{a \Delta \varepsilon_{\mathrm{r}}^{0}}{\tilde{k}} J_{1}(\tilde{k} a)
$$

The integral is intractable if we wish to treat error function diffusion layers. Following (4.33), however, we estimate the cut-off due to both laminar and turbulent layers at the boundary of the oval by the following factor applied to (4.39):

$$
\mathrm{e}^{-\widetilde{k}^{2} \delta^{2} / 4}
$$

where $\delta$ is either $\delta_{\text {diff }}$ or $\delta_{\text {braids }}(\$ 2.3 .3)$.

\section{Numerical Results}

The next three sub-sections contain numerical results for the radar cross-sections of the $2 \mathrm{D}$ mechanisms discussed in $\$ 2$. Since at this time amplitudes and wavenumbers of $3 \mathrm{D}$ disturbances in the vortex cores are not known, plots for this mechanism are omitted.

\subsection{Scattering by $2 D$ density variations in a vortex}

The results of the full Born analysis were obtained using Method A ( $\$ 3.5)$ and a Gaussian beam having a half-width of $\theta_{\mathrm{b}}=1.5^{\circ}$ and angle of polarization $\gamma=0$ is assumed.

In (4.32) the width $\delta_{\varepsilon}$ of the well in dielectric constant was taken to be $.025 b_{0}$ and the depth $\Delta \varepsilon_{\mathrm{r}}^{0}$ of the well was taken to be $-6 \times 10^{-6}$ (both from figure 1).

Figure $4 a$ plots the radar cross-section at a range of $1 \mathrm{~km}$. The results of the approximate analysis (lines) agree quite well with those of the Born analysis (symbols) even though the far-field parameter becomes $\mathcal{O}(1)$ (Figure 4b). The general features of the curves are in accord with the discussion in $\$ 4.3 .1$. One observes (i) a low frequency cut-off for back-scattering away from normal incidence due to finite beam width (chain-dashed), (ii) a higher frequency cut-off at the scale of the vortex for back-scattering at normal incidence (dashed) and specular reflection (dotted), and (iii) elimination of both cut-offs for forward scattering (solid). The long-dashed curve shows that some increase in frequency and reflectivity can be obtained even somewhat away from the forward direction, in particular when the transmitter and receiver are both looking up at the vortex with an elevation of $30^{\circ}$.

The limit frequency for the Born approximation is obtained by setting $L_{\mathrm{s}}=\max \left(u_{\mathrm{b}}, \delta_{\varepsilon}\right)$ in (3.12). The result is $f \ll .74 \mathrm{GHz}$. This is only marginally satisfied for the rightmost portion of the figure. From the Mie result for scattering by a sphere we expect that if the Born approximation were not made, the main difference would be that the solid line, which increases line:r!y, would begin to oscillate and decrease in average'slope as the weak scattering app:oximation began to be violated.

To emphasize differences between the full Born and approximate analysis the range was varied beyond $1 \mathrm{~km}$. For a point target the radar cross-section should ba independent of range but not so for an extended target. For very short ranges the vortex will fill the beam and we expect the RCS to increase quadratically with range. When the range is large enough that the vortex fills the beam only in one direction then we expect that the radar-cross should increase linearly. To verify this, the frequency was kept fixed at the peak for normal back-scattering $\left(f=5 \times 10^{7} \mathrm{~Hz}\right)$ and the range was varied (see circles in figure 5a). For off-normal back-scattering the frequency was fixed at $f=2 \times 10^{6} \mathrm{~Hz}$. The square symbols in figure $5 a$ show that as the beam radius increases with range the cut-off becones sharper. It is interesting to note that the dependence on range according to the approximate analysis (dashed line) is quadratic, that is, as if the target were beam filling 

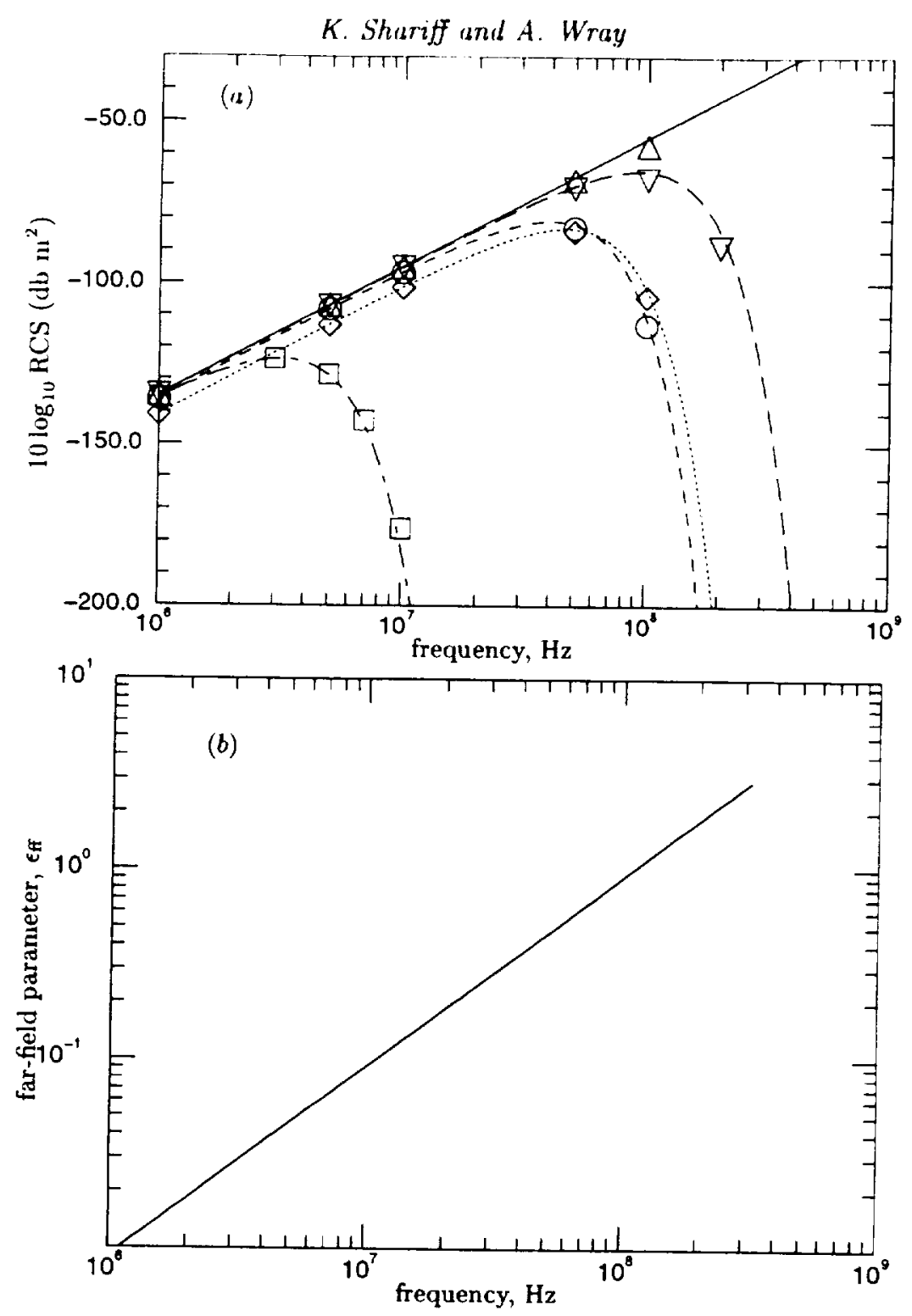

FIGURE 4. (a) Radar cross-section for a vortex due to a Gaussian beam at a range of $1 \mathrm{~km}$. The symbols correspond to the Born analysis while the lines give the result of the approximate analysis. - - , $\square$ : back-scattering away from normal incidence $\left(\alpha_{t}=\alpha_{r}=30^{\circ}, \beta_{t}=\beta_{r}=0\right)$; ,$--- \circ$ : back-scattering at normal incidence $\left(\alpha_{t}=\alpha_{r}=0^{\circ}, \beta_{t}=\beta_{r}=0\right) ; \cdots \cdots \cdots, 0$; specular reflection $\left(\alpha_{t}=-\alpha_{r}=30^{\circ}, \beta_{t}=\beta_{r}=0\right) ;---, \nabla:$ nearly forward scattering with $60^{\circ}$ offset in $\beta\left(\alpha_{t}=-\alpha_{r}=30^{\circ}, \beta_{t}=0, \beta_{r}=120^{\circ}\right)$.,$\Delta$ : forward scattering $\left(\alpha_{t}=-\alpha_{r}=30^{\circ}, \beta_{t}=0, \beta_{r}=180^{\circ}\right)$. (b) Far-field parameter, $\epsilon_{\mathrm{ff}}$ for the top figure.

in both directions. Figure $5 b$ shows that the approximate begins to show deviations when the far-field parameter becomes greater than unity.

\subsection{Scattering by the oval}

\subsubsection{Comparison with Gilson's (1992) Experiments}

Experimental procedures and parameters (Gilson 1992, Roth et al.1989) are now discussed: 

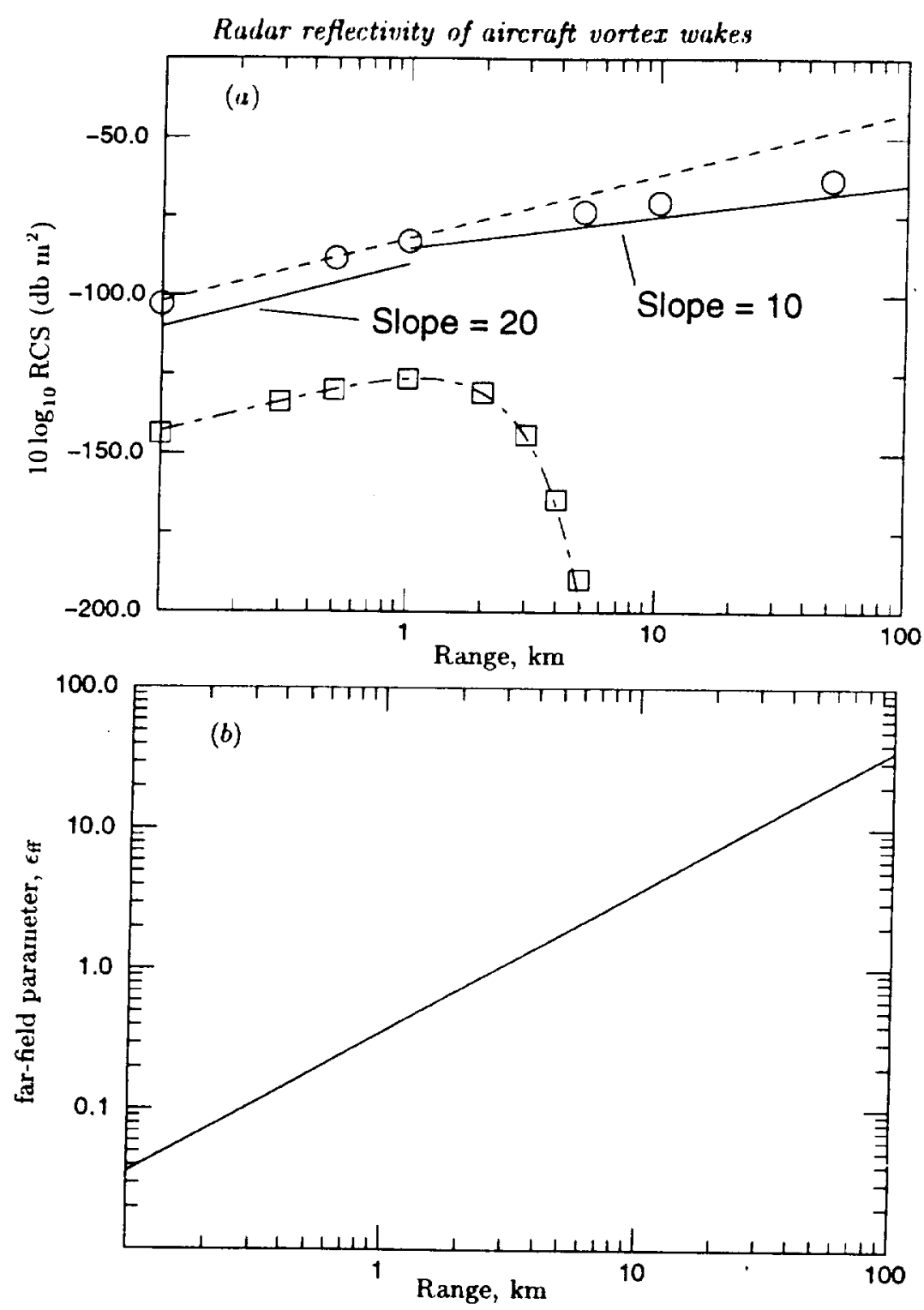

Figure 5. (a) Dependence of the radar cross-section of a vortex on range. The symbol$s$ correspond to the analysis with only the Born approximation while the broken lines give the result of the approximate analysis. - - , 0 : back-scattering at normal incidence $\left(\alpha_{t}=\alpha_{r}=0^{\circ}, \beta_{t}=\beta_{r}=0\right), f=5 \times 10^{7} \mathrm{~Hz} ;--$, : back-scattering away from normal incidence $\left(\alpha_{t}=\alpha_{r}=30^{\circ}, \beta_{t}=\beta_{r}=0\right), f=2 \times 10^{6} ;-$, reference lines to indicate quadratic and linear dependence. (b) Far-field parameter, $\epsilon_{\mathrm{ff}}$ for the top figure.

(i) Gilson (1992) reports RCS data in $\mathrm{db} \mathrm{m}^{2}$ per meter of wake length (Gilson 1992, his p. 37) along the radar beam. Hence, we multiplied his RCS values by our estimate $(101.5 \mathrm{~m})$ for the diameter of the oval. This value is consistent with the size of the wake in the radar returns.

(ii) The range of the target was $15 \mathrm{~km}$ and we chose the case having the lowest flight altitude (5000 ft) presented in Gilson (1992). The radar was pointed normal to the wake.

(iii) All the radars in the experiment transmit a right circularly polarized (RCP) wave and receive both right and left circular polarized (LCP) waves. So far our analysis has 


$\begin{array}{cccccccc}\text { Band } & \Delta \theta & \theta_{\mathrm{b}} & f_{0} & f_{\text {lim }} & \text { Waveform } & \text { Pulse width, } & \text { F.M spread, } \\ & \text { (mrads) } & \text { (degrees) } & (\mathrm{GHz}) & (\mathrm{GHz}) & \text { ID } & \tau(\mu \mathrm{s}) & \Delta f_{F . M}(\mathrm{MHz}) \\ \text { VHF } & 48.3 & 2.37 & 0.162 & 0.084 & V .25 \mathrm{C} & 0.25 & 0 \\ \mathrm{UHF} & 19.2 & 0.934 & 0.422 & 0.21 & \mathrm{U} .1 \mathrm{C} & 0.10 & 0 \\ \mathrm{~L} & 10.6 & 0.516 & 1.32 & 0.39 & \mathrm{~L} 2 & 2 & 20 \\ \mathrm{~S} & 5.2 & 0.253 & 2.35 & 0.79 & \mathrm{~S} 3 & 3 & 60 \\ \mathrm{C} & 5.2 & 0.253 & 5.67 & 0.79 & \mathrm{NB} & 10.2 & 6 \\ \mathrm{~K}_{\mathrm{a}} & 0.76 & 0.037 & 35 & 1.0 & \mathrm{~N} 2 & 50 & 12\end{array}$

TABLE 2. Gilson's radar parameters. $\Delta \theta$ : half-power beamwidth; $\theta_{\mathrm{b}}: \mathrm{e}^{-1}$ half-width of a Gaussian beam; $f_{l i m}$ : Limit frequency for validity of the Born approximation.

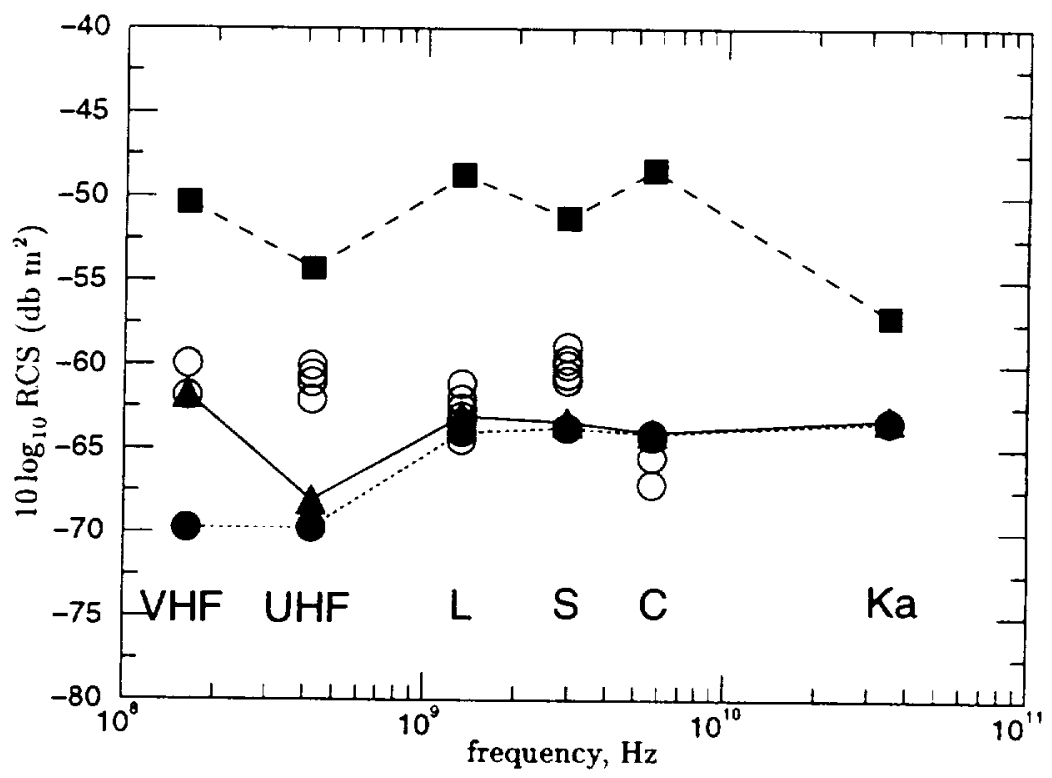

Figure 6. Comparison with Gilson's (1992) experiment. o, Gilson's data at 66 secs behind the plane; -....... : Full Born analysis for oval;,- : Full Born analysis for oval with a slice of entrained fluid. - --- : Approximate analysis for the oval.

been for linear polarization. However, a CP wave is simply a superposition of two linearly polarized waves

$$
\begin{aligned}
\mathbf{E}(\mathbf{x}) & =E(\mathbf{x})\left(\widehat{\mathbf{e}}_{1} \pm \mathrm{i} \widehat{\mathbf{e}}_{2}\right), \\
\mathbf{H}(\mathbf{x}) & =H(\mathbf{x})\left(\widehat{\mathbf{e}}_{1} \pm \mathrm{i} \widehat{\mathbf{e}}_{2}\right),
\end{aligned}
$$

where $\widehat{\mathbf{e}}_{1} \perp \widehat{\mathbf{e}}_{2}$. First note that the power flux of a $\mathrm{CP}$ wave is simply twice the power flux of one of its linearly polarized components. If the incident beam has the form (5.2) then the linearly polarized analysis can be done separately for each component and the resulting scattered fields added. In this manner, it was verified that for all the cases presented here, the resulting scattered wave is also CP (but of opposite helicity). Thus both incident and scattered powers are doubled compared to the linearly polarized case and the RCS is left unchanged.

(iv) Gilson used a pulsed radar whereas our analysis is for a time-harmonic wave. Appendix B describes the treatment of a pulse and Table 5.2.1 gives the characteristics of the waveforms. 


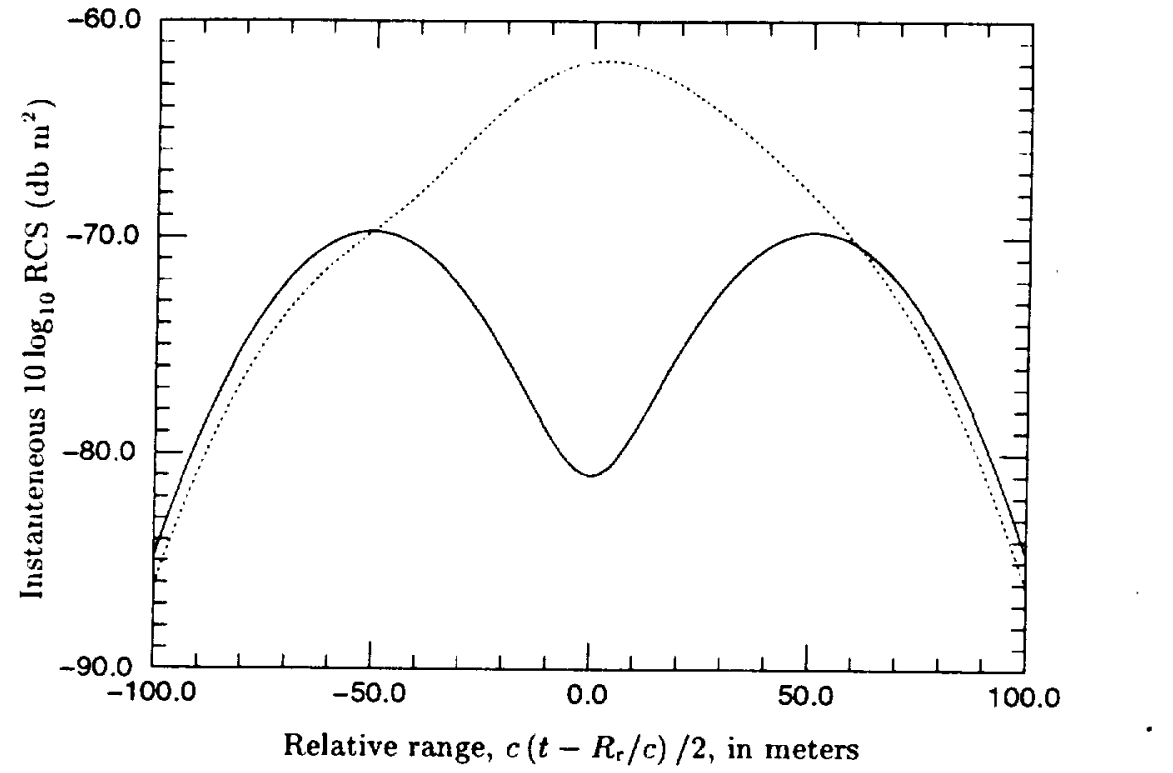

FIGURE 7. Returned pulse envelope calculated for Gilson's (1992) VHF case. The abcissa is time expressed as a range, $c\left(t-R_{r} / c\right) / 2$; the division by 2 converts from round-trip travel distance between scattering centers to one-way distance. - : Oval; $\cdots \cdots . . .$. : Oval with slice of entrained ambient fuid.

Figure 6 compares predicted and measured RCS values. The open circles are measurements 66 secs behind the plane. The predictions of the full Born analysis for the oval are the solid circles. Each value plotted represents the peak value of $\operatorname{RCS}(t)$ in the returned pulse. The predictions agree with the measurements for bands $L, S$, and $C$.

At VHF and UHF, the pulse widths are so short that in the calculation, the two scattering centers of the target (see discussion after Eq. B 14 in Appendix B) produce two distinct echoes (see solid line in figure 7). If the electric field of the two echoes are added, or if a long pulse is used such that the two echoes overlap, then the result matches the experiment. Such addition can only be justified if signal processing in the experiment added returns from multiple range gates within the wake but according to $W$. Gilson (Private communication) this was not so. Addition could also occur if there are, in reality, more than two scattering centers in the target spaced sufficiently close to each

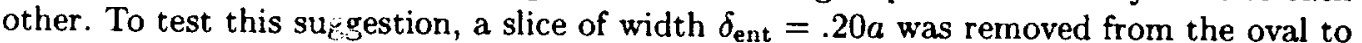
represent entrained anbient fluid (figure 8). With elevation angle $\beta=5.8^{\circ}$, the value for Gilson's (1992) experiment, the resulting echo, shown as the dotted line in figure 7 now consists of one main pulse. The RCS predictions are shown as the solid triangles in figure 6 . The agreement at VHF has now improved but not at UHF which has a very narrow pulse width. It is possible that the target still has more internal structure we have not accounted for.

Gilson obtained no return at $\mathrm{k}_{n}$ band $(35 \mathrm{GHz})$. Figure 9 shows the attenuation in RCS, due to the oval boundary estimated using (4.40). For the thickness of the turbulent layer the result (solid line) fits observations: there is very little attenuation at $5.66 \mathrm{GHz}$ but a lot at $35 \mathrm{GHz}$. The thicker laminar layer, however, attenuates too strongly (dotted line).

An upper frequency limit $f_{\text {lim }}$ for validity of the Born approximation was defined using 


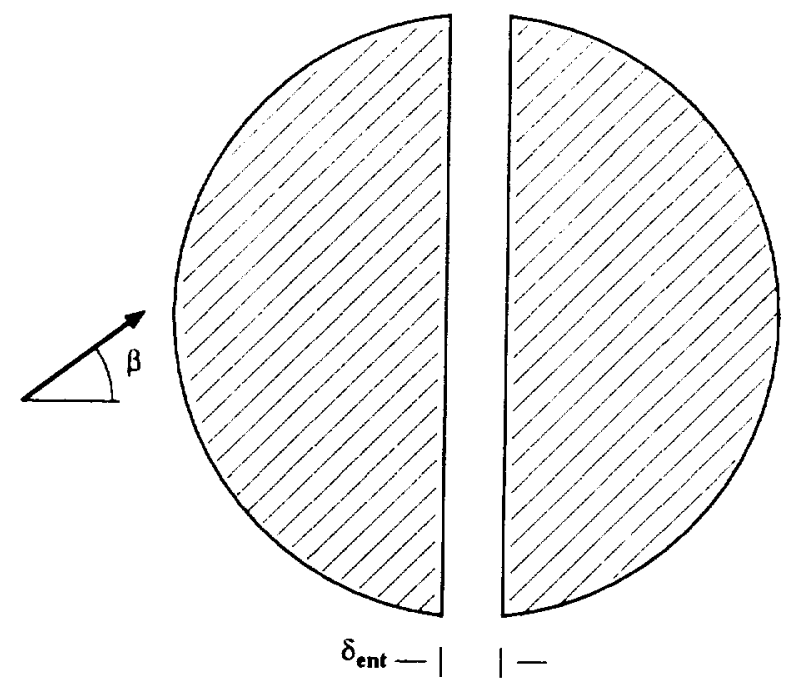

Figure 8. Model for the oval with a slice of entrained ambient fluid.

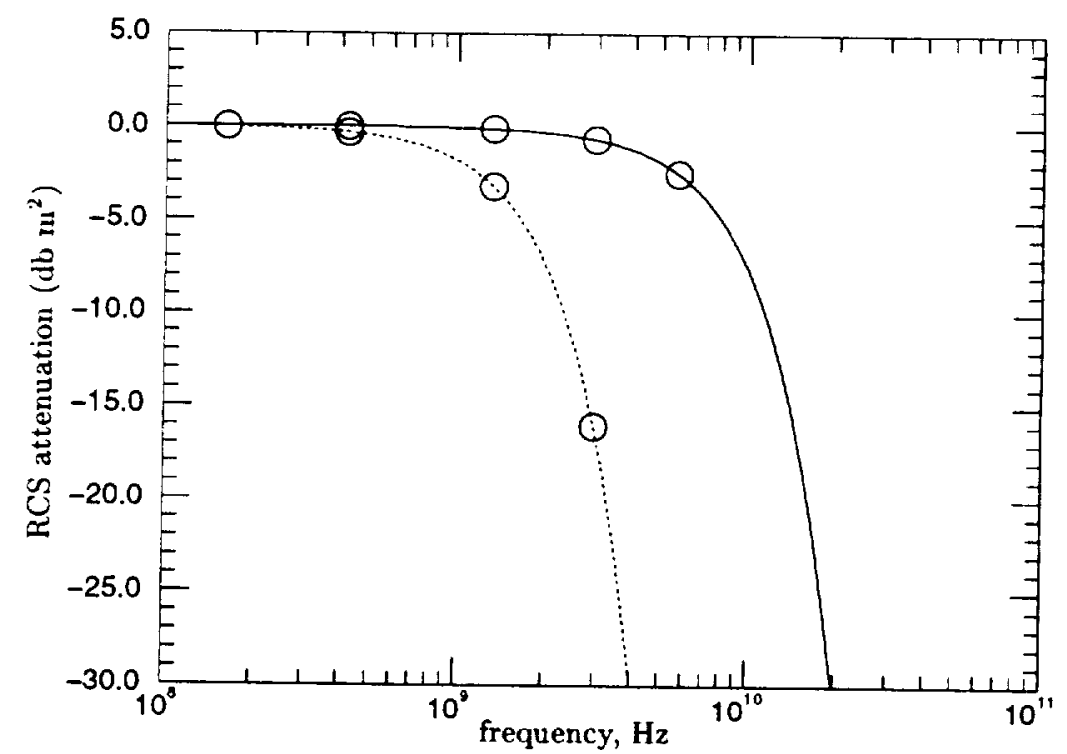

FIGURE 9. RCS attenuation due to the oval boundary. $\delta=\delta_{\text {braids }}=0.45 \mathrm{~cm} ; \circ$ : Frequencies corresponding to Gilson's experiment.

(3.12) by replacing the inequality with an equality and using

$$
L_{\mathrm{s}}=\max \left(R_{t} \sin \left(\theta_{\mathrm{b}}\right), a\right)
$$

for the characteristic radius of the scattering region. Table 5.2.1 shows that Gilson's experiment has frequencies between factors of 2 and 6 above $f_{\text {lim. }}$. Therefore in the future it would be worthwhile to consider another approach such as geometric optics which valid for large $k a$ but arbitrary $\Delta \varepsilon_{\mathrm{r}}$.

The analysis predicts a rapid cut-off away from normal incidence. On the other hand, comparing experiments at $45^{\circ}$ and $90^{\circ}$ incidence (Gilson's runs 1 and 17 , respectively) does not reveal significant differences. Moreover, Gilson searched for a flash at normal 


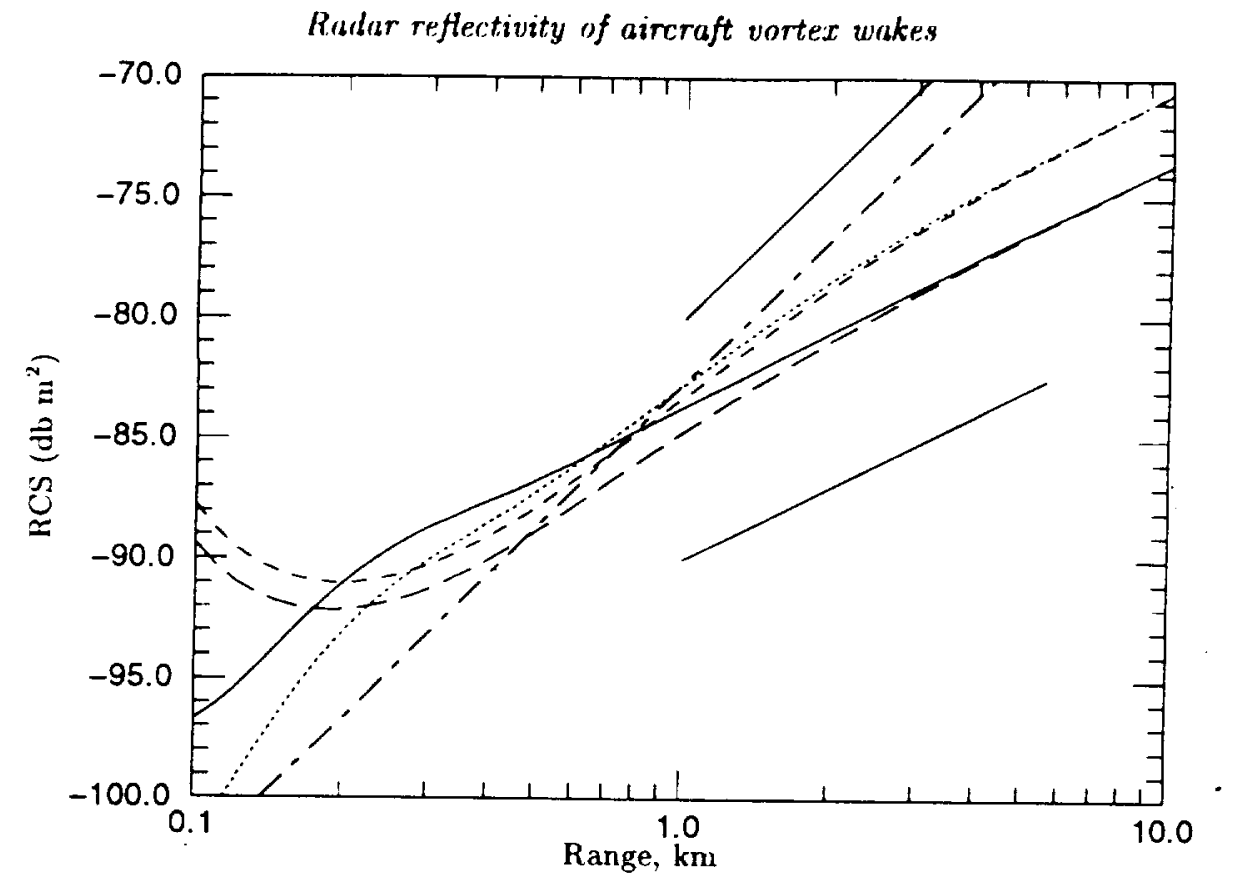

FIGURE 10. Test of various approximations. —— : full Born analysis; $\cdots \cdots . .$. : with the far-field Green's function; --- : with the wide cylindrical beam approximation; - - : : with both approximations. - - : same as ine wide cylindrical beam approximation but without assumption (d) in Appendix A.2. The thick lines depict linear and quadratic dependence on range.

incidence and found none. We estimated in $\S 2.3 .3$ that the boundary of the oval is very likely to be turbulent and this may account for the lack of directional dependence in the experiment.

\subsubsection{Test of various approximations}

Range dependence. The effect of various approximations was studied using Method $B$ by providing the option to use either the far-field or the full Green's function and the option to use the either the conical beam or the approximate cylindrical beam. Figure 10 shows the results. The parameters chosen were those of Gilson's S-band test except that the range was varied. The result (solid line) of the full Born analysis shows the $R^{2}$ behavior for small ranges, characteristic of a beam filling target, followed bv the linear behavior characteristic of a target that fills the beam in only one direction. As the range increases both the far-field $G$ (dotted) and approximate beam (dashed) results seem to agree with each other and to overpredict the RCS of the full Born analysis'(solid). This agreement partially understandable since both require smallness of a far-fieid parameter (see approximation (d) in Appendix A.2) in order to be valid. If we drop assumption (d), the remaining assumptions ((a)-(c) in Appendix A.2) should begin to hold as the range increases and the long dashed curve bears this out. When both the far-field $G$ and approximate beam are used, the result (chain-dashed) gives an $R^{2}$ behavior throughout which is erroneous for large $R$. This curve agrees, as it should, with the result of the approximate analysis; the comparison is not shown for clarity.

Frequency dependence. Figure 11 shows the frequency dependence of the RCS of the oval for a time harmonic incident wave. The rest of the parameters are those for Gilson's VHF case and his data (circles) has been included for reference even though they are strictly for a pulse. The solid line is the result of the full-Born analysis and shows a flat frequency dependence. The curve is punctuated by oscillations. These oscillations have 


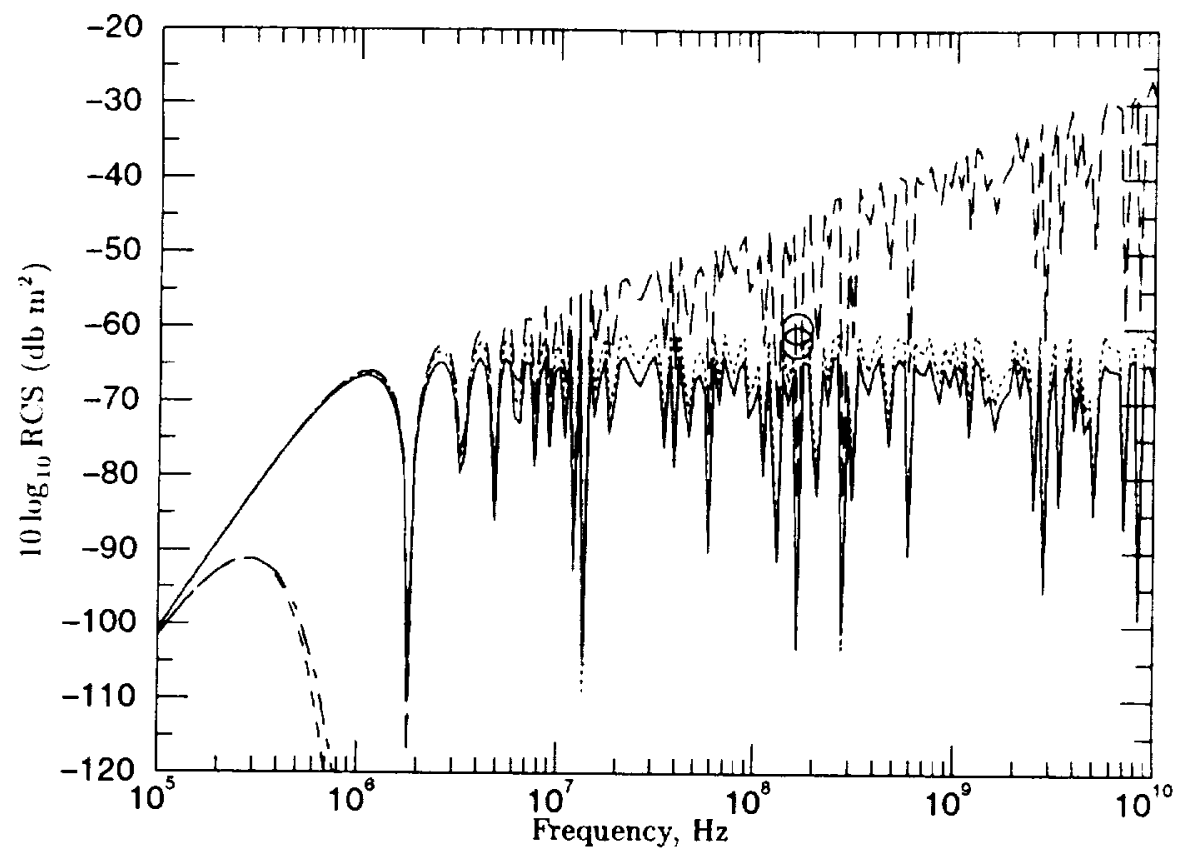

FIGURE 11. Frequency dependence of the RCS for the oval. — : full Born analysis (normal incidence); - - : approximate analysis (normal incidence); $\cdots \cdots \cdot$ : far-field $G$ (normal inci-

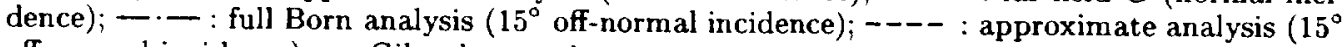
off-normal incidence); $\circ:$ Gilson's experiment.

a regular period (versus frequency) of $\Delta f=c /(4 a)$ due to interference between the two effective scattering centers of the target (see Appendix B). Except for the lowest frequency decades, the number of plot points is so coarse that the fluctuations appear irregular. Using the far-field $G$ produces the dotted curve which only slightly overpredicts the RCS. The curve for the wide cylindrical beam is very close to the dotted curve and is not shown for clarity. However, when both far-field $G$ and wide cylindrical beam are invoked (to yield the approximate analysis) the result (long-dashed curve) has an erroneous RCS $\propto k$ behavior. For the same number of plot points, the dotted and long-dashed curves have the same pattern of oscillations indicating that the period and phase of the oscillations is the same; only the amplitude is higher. The other two curves with the sharp cut-off at lower-left are for off-normal incidence $\left(\alpha_{r}=\alpha_{t}=15^{\circ}\right)$.

\section{Practical Recommendations}

\subsection{Prospects for detection of the vortex cores}

No test so far has been at frequencies as low as $49 \mathrm{MHz}$ where, according to the analysis, the density gradient in the vortex cores reflects (see dashed line in figure 4). Since this mechanism is independent of atmospheric conditions, it is worthwhile to discuss its potential use in air traffic control (ATC).

(i) It is fortunate that $49 \mathrm{MHz}$ is where many radars operate around the world for atmospheric profiling. Such radars are called ST or MST radars and they employ an antenna array. Thus the predicted reflectivity could very well be tested. be.

(ii) Rain clutter is not a problem at this frequency, though clear air turbulence might

(iii) Most ST radars are used for measurements in the high atmosphere but recently 
various problems associated with measurements at lower heights have been overcome and a boundary layer profiler has been developed (Vincent et al.1998) which has a height coverage of $400-3200 \mathrm{~m}$. This radar achieves a range resolution of $150 \mathrm{~m}$ with a pulse width of 1 lus.

(iv) For ATC use, the required cross bean resolution (beamwidth) should be a fraction of the minimum airplane spacing expected (about 2 nautical miles). The smaller the beamwidth the larger the required array but, since the antenna gain is larger, less power is required. Desiring both modest array size and modest power we impose a cross beam resolution of $0.3 \mathrm{n.m}$. at ranges $<3 \mathrm{~km}$ resulting in a $10 \times 10$ array with elements spaced $3 \mathrm{~m}$ apart and a power of only $360 \mathrm{~W}$. Since this value is surprisingly modest, details of the calculation follow.

For an antenna array the half-power beamwidth (in degrees) broadside to the array is (Skolnik 1970, p. 11-2):

$$
\Delta \theta_{1 / 2} \approx \frac{100}{\sqrt{N_{\mathrm{el}}}}
$$

where $N_{\mathrm{el}}$ is the number of elements, and the gain on transmit and receive is: $G_{\mathrm{t}}=G_{\mathrm{r}} \approx$ $\pi N_{\mathrm{el}}$. The transmitter power required for detection is:

$$
P_{\mathrm{t}}=\frac{(4 \pi)^{3} R^{4} N_{\mathrm{p}} \times \mathrm{SNR}}{G_{\mathrm{t}} G_{\mathrm{r}} \lambda^{2} L_{\text {sys }} \times \mathrm{RCS}}
$$

where $N_{\mathrm{p}}=-185 \mathrm{db} W$ and $L_{\text {sys }}=-7 \mathrm{db}$ are the noise power and system loss for an ST radar (Kingsley \& Quegan 1992, p. 199). SNR $=20 \mathrm{db}$ is the desired signal to noise ratio, $\lambda=6 \mathrm{~m}$ is the wavelength and $\mathrm{RCS}=-77 \mathrm{db}$, a value we calculated for a vortex at $3 \mathrm{~km}$ using the beamwidth of the $10 \times 10$ array.

(v) ST radars have limited steering capability, usually just a few fixed beam directions but this may be sufficient to observe the approach path of a few adjacent runways at a fixed distance from the touch-down point. Since it is unlikely that three dimensional disturbances will exist in the vortex across a broad spectrum of wave angles, detection may be possible at only normal incidence.

(vi) Finally let us note that the oval will also scatter at $49 \mathrm{MHz}$.

The above estimat es have ignored smaller aircraft types and detection of vortices of aircaft in landing connfigurations. Such considerations would have to be taken into account in a full-fledged feasibility study.

\subsection{A note on the role of engine exhaust}

At approach, the exhaust of a large aircraft has a temperature of $477 \mathrm{~K}$ and each engine emits $1200-6000 \mathrm{lb} / \mathrm{hr}$ of water vapor (P. Spalart, Private communication). If we consider the temperature cortribution alone (the first term in Eq. 2.1) we get $\Delta \varepsilon_{\mathrm{r}}=-2.1 \times 10^{-4}$. This is roughly two orders of magnitude greater than for the density variation in each vortex and for atmospheric density stratification and would lead to a $40 \mathrm{db}$ greater radar cross-section. However, Gilson (1992) found that the wake RCS at $1 \mathrm{~km}$ behind the plane did not change when the engines were run at idle or full power. This is probably due to turbulent mixing of the exhaust with the ambient. Diversion of some engine exhaust into the laminarising flow of the vortex cores may allow the exhaust to maintain its heat further downstream.

A simpler alternative is to detect the exhaust itself as in the Noonkester \& Richter (1980) experiment. Using the radar trace of the exhaust spiral (their figure 5) we deduced that the circulation of the vortices is $400 \mathrm{~m}^{2} / \mathrm{s}$. In clear weather, an even simpler alternative is to view the exhaust. spiral in infrared in order to deduce the vortex circulation. 
To estimate the contribution of water vapor in the exhaust, use the upper value of $6000 \mathrm{lb} / \mathrm{hr} /$ engine and assume an exhaust diameter of $2 \mathrm{~m}$. This yields a partial pressure of vapor equal to $1.1 \mathrm{mb}$ and a contribution of $\Delta \varepsilon_{\mathrm{r}}$ of $3.7 \times 10^{-6}$ which is much smaller than that due to temperature.

\section{Conclusions}

(i) The measured radar cross-sections in the experiments of Gilson generally agree with the values caclulated using the simple picture of a oval carrying atmospheric fund as it descends. The lack of directional dependence is not predicted by the analysis, however, estimates indicate that this is because the boundary of the oval is very likely turbulent.

(ii) The density gradient in the vortices themselves produces index of refraction variations comparable to those produced by the oval. This provides a scattering mechanism independent of atmopsheric consitions and could be detected using an ST type radar at about $49 \mathrm{MHz}$.

(iii) The use of a far-field Green's function is not a priori valid for a wake that is coherent along its length and therefore a more general analysis was developed. The use of a far-field $G$ was found to produce a constant db error in the radar cross-section for large ranges. The neglect of beam conicality produces similar errors. When both assumptions are made, the dependence of radar cross-section on range is altered from the correct linear behavior (as expected for a target that fills the beam in one direction) to quadratic behavior. Similarly, the frequency dependence is altered from being flat to $\propto k$.

We thank Drs. W. Gilson and P. Ingwersen (MIT Lincoln Labs.) for providing us with information on the experiment, Dr. P. Spalart (Boeing) for suggesting that we account for density variation inside a vortex core and for supplying the information referred to in the text. Thanks are also due to Prof. J. Jimenez (Univ. Madrid) for suggesting the inclusion of water vapor in the refractive index and to Dr. M. Wang (CTR) for help with integrals.

\section{Appendix $\mathbf{A}$. The electric field in the incident beam: $\mathbf{E}_{\mathrm{i}}(x, y, z)$}

The incident electric field is obtained using aperture antenna analysis (Silver 1949) where one derives the far diffraction field from knowing the field on a surface (the "aperture") near the antenna. We shall take the aperture to be circular.

For convenience we shall, in the manner of a subroutine, use a dummy coordinate system $(x, y, z)$ centered on the aperture. The plane of the aperture is $x y$ and $z$ is normal to the aperture. These coordinates should not be confused with those in the body of the paper where they refer to wake coordinates and where $\left(x^{*}, y^{*}, z^{*}\right)$ refer to aperture coordinates.

\section{A.1. Conical beam}

According to Silver (1949), the electric field created in the far zone by a plane aperture $A$ on which the electric field is specified to be $\mathbf{E}=E_{x}^{a}\left(\mathbf{x}^{\prime}\right) \hat{\mathbf{x}}$ is the following integral over the aperture (Silver 1949, p. 167, his Eq. 124):

$$
\mathbf{E}_{\mathrm{i}}(\mathbf{x})=\frac{i k}{4 \pi r} \widehat{\mathbf{x}} \mathrm{e}^{\mathrm{i} k r}(1+\cos \theta) \int_{A} E_{x}^{a}\left(\mathbf{x}^{\prime}\right) \mathrm{e}^{\mathrm{i} k \mathbf{x}^{\prime} \cdot \mathbf{x} /|\mathbf{x}|} d \mathbf{x}^{\prime} .
$$


Here $(r, \theta)$ are the spherical polar coordinates of $\mathbf{x}, \theta$ being the polar angle measured from the $z$ axis. Equation (A 1 ) is derived starting from vector Kirchhoff integrals and introducing several approximations valid in the limit of small wavelength compared to aperture diameter in which case the signiticant portion of the energy is confined to small $\theta$. In addition, the phase of the field on the aperture has been assurned to be constant.

For an axially symmetric distribution of $E_{x}^{a}$ on the aperture the integral in (A 1) becomes

$$
I_{A}=2 \pi \int \varrho^{\prime} d \varrho^{\prime} E_{x}^{a}\left(\varrho^{\prime}\right) \cdot J_{0}\left(k \varrho^{\prime} \sin \theta\right)
$$

where $\varrho^{\prime}=\sqrt{x^{\prime 2}+y^{\prime 2}}$. Two aperture illuminations are considered. For the case of a uniform illumination:

we get

$$
E_{x}^{a}(\varrho)= \begin{cases}E_{0}, & \varrho \leqslant \varrho_{a} \\ 0, & \text { otherwise }\end{cases}
$$

$$
I_{A}=2 \pi E_{0} \varrho_{a}^{2} \frac{J_{1}\left(k \varrho_{a} \sin \theta\right)}{k \varrho_{a} \sin \theta}
$$

For the case of Gaussian illumination of the aperture

$$
E_{x}^{a}(\varrho)=E_{0} \mathrm{e}^{-\left(\varrho / \sigma_{a}\right)^{2}}
$$

we get

$$
I_{A}=\pi \sigma_{a}^{2} E_{0} \exp \left(-\frac{1}{4} k^{2} \sigma_{a}^{2} \sin ^{2} \theta\right)
$$

thanks to an integral tabulated in Gradshteyn \& Ryzhik (1980, p. 717,6.63.4) To calculate the radar cross-section according to (3.13) requires the maximum value at the target of the time averaged power flux $S_{i}=1 / 2 \operatorname{Re}\left\{E_{i} \times H_{i}^{*}\right\}$, where $H_{i}$ can be obtained from the Maxwell equation $\mathbf{H}_{\mathbf{i}}=1 /\left(i \omega \mu_{0}\right) \nabla \times \mathbf{E}_{\mathbf{i}}$. If in evaluating $\nabla \times \mathbf{E}_{\mathbf{i}}$ from (A 1) we retain only the leading order term $(\mathcal{O}(1 / r))$ and introduce $\mu_{0}=1 /\left(c^{2} \varepsilon_{0}\right)$ we get

$$
\mathrm{S}_{\mathrm{i}}=\frac{1}{2} c \varepsilon_{0}\left(E_{x}^{\mathrm{i}}\right)^{2} \frac{z}{r} \widehat{\mathbf{z}}
$$

where $E_{x}^{\mathbf{i}}$ denotes the $x$ component of $\mathbf{E}_{\mathbf{i}}$. The radar cross-section is independent of the amplitude $E_{0}$ since both incident and scattered powers are proportional to $E_{0}^{2}$. For convenience, we chose

$$
E_{0}=\left\{\begin{array}{ll}
2 R_{t} /\left(i k \varrho_{a}^{2}\right), & \text { for uniform aperture illumination, } \\
2 R_{t} /\left(i k \sigma_{a}^{2}\right), & \text { for Gaussian aperture illumination, }
\end{array},\right.
$$

in order to make equal to $\mathrm{e}^{\mathrm{i} k R_{\mathrm{t}}}$ the maximum value of $E_{x}^{\mathrm{i}}$ with respect to $\theta$ at fixed $r=R_{t}$. The denominator in (3.13) is then

$$
\text { maximum power density in incident beam at target }=\frac{1}{2} c \varepsilon_{0} \text {. }
$$

In the calculations, instead of specifying the aperture radius as the input parameter, we specify the half beanwidth $\theta_{\mathrm{b}}$ and from it calculate the implied value of $\varrho_{a}$. For the Gaussian beam $\theta_{\mathrm{b}}$ is defined to be where $I_{A}$ drops to $\mathrm{e}^{-1}$ of its peak value and for the Bessel beam it is defined to be the location of the first zero thus giving

$$
\varrho_{a}=\frac{3.83171}{k \sin \theta_{b}}, \quad \sigma_{a}=\frac{2}{k \sin \theta_{b}}
$$




\section{A.2. Approximate cylindrical beam}

The cylindrical beam for the approximate scattering analysis in $\$ 4$ of the paper is obtained after introducing four approximations in (A 1): (a) Replace $\cos \theta$ by 1. This is valid for a small angular beamwidth. (b) Replace $r$ in the denominator by a constant range $R_{t}$. (c) Sirnilarly, in (A 2) replace $\sin \theta=u / r$ by $u / R_{t}$, where $u=\sqrt{x^{2}+y^{2}}$ is the normal distance from the beam axis. (d) Replace $\mathrm{e}^{\mathrm{ikr}}$ with $\mathrm{e}^{\mathrm{i} k z}$. Approximations (b)-(c) are valid when the relative change in beam width across the near and far ranges of the target is small. For approximation (d), however, a condition similar to the the far-field condition (3.1) must hold:

$$
\frac{\pi L_{\mathrm{s}}^{2}}{\lambda R_{t}} \ll 1 .
$$

Introducing the stated approximations into (A 1$)$ gives:

$$
\mathbf{E}=\frac{i k}{2 \pi R_{t}} \hat{\mathbf{x}} \mathrm{e}^{\mathrm{i} k z} I_{A},
$$

Substituting (A 4) and (A 6) for $I_{A}$ into (A 12), replacing $\sin \theta$ with $u / R_{t}$, using (A 10), defining $u_{\mathrm{b}} / R_{t}=\sin \theta_{\mathrm{b}}$, and substituting values of $E_{0}$ from (A 8) we get

$$
\begin{aligned}
\mathbf{E} & =\widehat{\mathbf{x}} \mathrm{e}^{i k z} \frac{2 J_{1}(u / \sigma)}{u / \sigma}, \\
\sigma & \equiv u_{\mathrm{b}} / 3.8371
\end{aligned}
$$

for the Bessel function beam and

$$
\begin{aligned}
& \mathbf{E}=\widehat{\mathbf{x}} \mathrm{e}^{i k z} \exp \left(-u^{2} / \sigma^{2}\right) \\
& \sigma \equiv u_{\mathrm{b}}
\end{aligned}
$$

for the Gaussian beam. For the incident power flux in the cylindrical beam we use the same value, given by (A 9) as in the original conical beam.

\section{Appendix B. Scattering by a pulse}

Since the scattering problem is linear, treatment of a pulse is simply a matter of Fourier superposition of the results of the time-harmonic analysis. The analysis was performed for back-scattering at normal incidence.

We begin by reducing the originally linear vector problem to a linear scalar problem. This will provide the set-up for using formulae already derived in Ishimaru (1978).

For the time harmonic analysis the incident electric field (Eq. A 1) has the form:

$$
\overline{\mathbf{E}}_{\mathrm{i}}(\mathbf{x}, t ; \omega)=\widehat{\mathbf{x}} \operatorname{Re}\left[F_{\mathrm{i}}(\mathbf{x}, \omega) \mathrm{e}^{-\mathrm{i} \omega t}\right] .
$$

As in Appendix $A$ we are using $\mathbf{x}$ to denote aperture coordinates. For the accompanying magnetic field we assume the same spatial dependence:

$$
\overline{\mathbf{H}}_{\mathrm{i}}(\mathbf{x}, t ; \omega)=c \varepsilon_{0} \hat{\mathbf{y}} \operatorname{Re}\left[F_{\mathrm{i}}(\mathbf{x}, \omega) \mathrm{e}^{-\mathrm{i} \omega t}\right] .
$$

According to Maxwell's equations $\overline{\mathbf{E}}$ is related to the curl of $\overline{\mathbf{H}}$ and therefore $\overline{\mathbf{E}}$ and $\overline{\mathbf{H}}$ should in general have different spatial dependence. However, to the extent that the wavelength is much smaller than the beamwidth, derivatives of $F(\mathbf{x}, \omega)$ normal to the beam axis will be much smaller than derivatives along the beam axis and the forms ( $B$ 1) and (B 2) will nearly satisfy Maxwell's equations and be divergence free.

Henceforth we will write the incident field as a function of time only, taking it to be 
evaluated at the point $\mathbf{x}=\left(0, y_{0}, 0\right)$ where the bearn centerline intersects the centerplane of the target. By a Fourier synthesis of (B 1) and (B 2) in time an arbitrary $L_{2}$ time dependence can be constructed:

$$
\begin{aligned}
\overline{\mathbf{E}}_{\mathrm{i}}(t) & =\widehat{\mathbf{x}} \operatorname{Re}\left[u_{\mathrm{i}}(t) \mathrm{e}^{-\mathrm{i} \omega_{n} t}\right], \\
\overline{\mathbf{H}}_{\mathrm{i}}(t) & =c \varepsilon_{0} \hat{\mathbf{y}} \operatorname{Re}\left[u_{\mathrm{i}}(t) \mathrm{e}^{-\mathrm{i} \omega_{0} t}\right],
\end{aligned}
$$

where we have chosen to write the time dependence as the product of a carrier $\mathrm{e}^{-\mathrm{i} \omega_{0} t}$ and a complex modulation $u_{\mathrm{i}}(t)$. The complex modulation is itself written as a product:

$$
u_{\mathrm{i}}(t)=f(t) \mathrm{e}^{\mathrm{i} \phi(t)},
$$

where $f(t)$ and $\phi(t)$ are real and describe amplitude and phase modulation, respectively.

We now assume that for the time harmonic analysis the scattered field evaluated at the receiver is also of the form (B 1) and (B 2) with $F_{\mathrm{i}}$ replaced by $F_{\mathrm{s}}$. This assumption was verified to be true from the numerical results. Therefore by Fourier superposition we can write for arbitrary time dependence:

$$
\begin{aligned}
\overline{\mathbf{E}}_{\mathbf{s}}(t) & =\widehat{\mathbf{x}} \operatorname{Re}\left[u_{\mathrm{s}}(t) \mathrm{e}^{-\mathrm{i} \omega_{0} t}\right], \\
\overline{\mathbf{H}}_{\mathbf{s}}(t) & =c \varepsilon_{0} \hat{\mathbf{y}} \operatorname{Re}\left[u_{\mathrm{s}}(t) \mathrm{e}^{-\mathrm{i} \omega_{0} t}\right],
\end{aligned}
$$

Our incident and scattered fields thus depend only on the scalar functions $u_{\mathrm{i}}(t)$ and $u_{\mathrm{s}}(t)$ and we are thus set-up to use Ishimaru's (1978, vol. 1, p. 94) discussion for scattering by a pulse. Using the fact that scattering is a linear and causal problem, Ishimaru derives the following "input/output" relation for the complex modulation of the scattered pulse in terms of the modulation of the incident pulse:

$$
u_{\mathrm{s}}(t)=\int_{-\infty}^{\infty} U_{\mathrm{i}}(\omega) H\left(\omega+\omega_{0}, t\right) \mathrm{e}^{-\mathrm{i} \omega t} d \omega
$$

where

$$
U_{\mathrm{i}}(\omega)=\frac{1}{2 \pi} \int_{-\infty}^{\infty} u_{\mathrm{i}}(t) \mathrm{e}^{\mathrm{i} \omega t} d t
$$

$H(\omega, t)$ has the interpretation that if $u_{\mathrm{i}}(t)=1$ (i.e. we have the time harmonic case) then $u_{s}(t)=H\left(\omega_{0}, t\right)$. In the time harmonic analysis we chose the incident field to have a normalization such that $u_{\mathrm{i}}(t)=\mathrm{e}^{\mathrm{i} k R_{t}}$. Hence $H\left(\omega_{0}, t\right)$ is simply the $u_{\mathrm{s}}$ we calculate in the time harmonic analysis divided by $\mathrm{e}^{\mathrm{i} k R_{\mathrm{t}}}$. Since we have considered a steady target, thus losing the doppler effect, $H\left(\omega_{0}, t\right)$ does not depend on time.

Let us obtain $H(\omega)$ implied by the result of the approximate analysis for the oval. For backscattering at normal incidence and using the asymptotic form of the Bessel function for large $k a$, Eqs. (4.30) and (4.39) give:

$$
\mathbf{E}_{\mathrm{s}}(\mathbf{x})=\widehat{\mathbf{x}} \frac{\mathrm{e}^{\mathrm{i} k\left(R_{\mathrm{t}}+R_{r}\right)}}{4 R_{r}}(k a)^{1 / 2} \sigma \Delta \varepsilon_{\mathrm{r}}^{0} \cos \left(2 k a-\frac{3}{4} \pi\right)
$$

The bandwidth of the pulse is typically small compared to the center frequency and so $k a$ can be considered to be constant when outside the cosine. Hence, over the bandwidth of the pulse

where

$$
H(\omega)=A \mathrm{e}^{\mathrm{i} k R_{r}} \cos \left(2 k a-\frac{3}{4} \pi\right), \quad k=\omega / c,
$$

$$
A=\frac{\Delta \varepsilon_{\mathrm{r}}^{0} \sigma\left(k_{0} a\right)^{1 / 2}}{4 R_{r}}
$$


is a real constant independent of $\omega$, and $k_{0}$ is the wavenumber corresponding to the center frequency. For the full Born analysis of the oval, it was found that $H(\omega)$ had a behavior approximated by (B 11) but with a different (and complex) value of $A$ which was obtained by fitting the form (B 11) to the numerically obtained results.

Substituting the form (B II) into the input/output relation (B 8) gives the complex modulation $u_{\mathrm{s}}(t)$ of the returned pulse. When this is substituted into (B 6) one obtains:

$$
\begin{array}{r}
\overline{\mathbf{E}}_{\mathrm{s}}(t)=\widehat{\mathbf{x}}|\cdot A| / 2\left\{f ( t _ { - } ^ { \prime } ) \operatorname { c o s } \left[\phi\left(t_{-}^{\prime}\right)-\omega_{0} t_{-}^{\prime}+\phi_{A}-\frac{3}{4} \pi\right.\right. \\
\left.f\left(t_{+}^{\prime}\right) \cos \left[\phi\left(t_{+}^{\prime}\right)-\omega_{0} t_{+}^{\prime}+\phi_{A}+\frac{3}{4} \pi\right]\right\}
\end{array}
$$

where

$$
t_{-}^{\prime}=t-\frac{R_{r}}{c}-\frac{2 a}{c}, \quad t_{+}^{\prime}=t-\frac{R_{r}}{c}+\frac{2 a}{c},
$$

$a$ is the radius of the oval, and $\phi_{A}$ is the phase angle of $A$. Equations (B 13) and (B 14) have the following interpretation. The returned pulse can be thought of as consisting of the incident pulse reflected twice, each time with reflection coefficient $A / 2$ and phase shifts $\phi_{A}= \pm 3 \pi / 4$. The two reflections occur at $z= \pm a$. The time shift $-R_{r} / c$ accounts for the travel time from the point $z=0$ to the receiver while the additional time shifts $\pm 2 a / c$ account for the round-trip time required to travel from $z=0$ and the two points $z= \pm a$.

For the oval with the slice of entrained ambient fluid, the behavior of $H(\omega)$ was more complicated than (B 11). In this case the procedure followed was to first calculate $H(\omega)$ for $\omega$ spanning an interval $\Delta \omega$ consisting of few pulse bandwidths about the center frequency. Actually a convenient auxiliary quantity $T(\omega)$ was calculated such that

$$
H(\omega, t)=\frac{T(\omega) \mathrm{e}^{i k R_{\mathbf{r}}}}{R_{\mathbf{r}}}
$$

Next $T(\omega)$ was multiplied by a Hanning window and Fourier analyzed such that:

$$
T(\omega)=\sum_{m} \widehat{T}_{m} \exp \left[\mathrm{i} \frac{2 \pi}{\Delta \omega} m\left(\omega-\omega_{0}\right)\right] .
$$

Knowing the coefficients $\widehat{T}_{m}$ one can obtain the complex modulation of the received signal by substituting (B 16) and (B 15) into (B 8):

$$
u_{\mathbf{s}}(t)=\frac{1}{R_{\mathrm{r}}} \sum_{m} \widehat{T}_{m} u_{\mathrm{i}}\left(t-\frac{R_{\mathrm{r}}}{c}-\frac{2 \pi m}{\Delta \omega}\right) .
$$

Rectangular and Gaussian forms of unit amplitude were implemented for the amplitude modulation:

$$
f(t)=\left\{\begin{array}{ll}
1, & |t| \leqslant \tau / 2, \\
0, & \text { elsewhere }
\end{array}, \quad f(t)=e^{-t^{2} / \tau^{2}} .\right.
$$

where $\tau$ is the pulse duration. In this paper only the results for the Gaussian pulse are plotted since the rectangular pulse gave very close RCS results (within $0.25 \mathrm{db}$ for a few spot checks). For FM a linear frequency variation (chirp) was considered:

$$
\phi(t)=\pi \alpha t^{2} .
$$

Within the pulse width, the spread $\Delta f_{F M}$ in the local frequency is

$$
\Delta f_{\mathrm{FM}}=|\alpha| \tau .
$$

The sign of $\alpha$ (up-chirp vs. down-chirp) was found to not matter. Table 5.2.1 gives $\tau$ and $\Delta f_{\mathrm{FM}}$ for Gilson's tests. 
The instantaneous power flux density vector associated with the incident pulse (B 3) and $(B+)$ is

$$
\overline{\mathbf{S}}_{\mathbf{i}}=\overline{\mathbf{E}}_{\mathbf{i}} \times \overline{\mathbf{H}}_{\mathbf{i}}=\hat{\mathbf{z}} c \varepsilon_{0} f^{2}(t) \cos ^{2}\left(\phi(t)-\omega_{0} t\right)
$$

Finally, the instantaneous scattered power flux density vector associated with (B6) and (B 7) is

$$
\overline{\mathbf{S}}_{\mathrm{s}}(t)=\widehat{\mathbf{z}} c \varepsilon_{0}\left|\mathbf{E}_{\mathrm{s}}(t)\right|^{2}
$$

From these we calculate an instantaneous RCS:

$$
\operatorname{RCS}(t)=4 \pi R_{r}^{2} \frac{\left|\overline{\mathbf{S}}_{\mathrm{s}}(t)\right|}{\left|\overline{\mathbf{S}}_{\mathrm{i}}\right|_{\max }}
$$

\section{REFERENCES}

Bardina, J., Ferziger, J. H. \& Rogallo, R. S. 1985 Effect of rotation on isotropic turbulence: computation and modeling. J. Fluid Mech. 154, 321-336.

Betchov, R. AND SzewczYK, A. 1963 Stability of a shear layer between parallel streams. Phys. Fluids 6, 1391-1396.

CAMPBell, S. D. et al. 1997 Wake vortex field measurement program at Memphis, Tennessee, Data Guide. NASA Contractor Rep. 201690.

CARlon, H. R. 1970 Infrared emission by fine water aerosols and fogs. Applied Optics 9, 20002006.

Chadwick, R. B., JoRdiv, J. \& DETMAN, T. 1984 Radar cross section measurements of wingtip vortices. Pror. ESA IGARSS 1984, vol. 1, pp. 479-483. Also published as ESA SP-215.

Crow, S. C. 1970 Stability theory for a pair of trailing vortices. AIAA J. 8, 2172-2179.

Gilson, W. H. 1992 Radar measurements of Aircraft Wakes. Project Report AAW-11, Lincoln Lab., MIT, Lexington, Mass.

GiLson, W. H. 1994 Aircraft wake RCS measurement. In NASA Contractor Rep. 10139, Part 2, pp. 603-623.

Gradshteyn, I. S. \& RYzhik, I. M. 1980 Table of Integrals, Series and Products Academic.

Handbook of Geophysics MacMillan.

HanNon, S. M. \& Thomson, J. A. 1994 Aircraft wake vortex detection and measurement with pulsed solid-state coherent laser radar. J. Modern Optics 41, 2175-2196.

IANNUZzELLI, R. J. et al. 1998 Aircraft wake detection using bistatic radar: analysis of experimental results. Johns Hopkins Appl. Phys. Lab. Tech. Digest 19, 299-314.

Ishimaru, A. 1978 Wave Propagation and Scattering in Random Media Academic.

JACKSON, J. D. 1962 Classical Electrodynamics John Wiley \& Sons.

KIngsleY, S. \& QUEGAN, S. 1992 Understanding Radar Systems McGraw-Hill.

KöPP, F. 1994 Doppler lidar investigation of wake vortex transport between closely spaced parallel runways. AIAA Journal 32, 805-810.

LANDMAN, M. J. \& SAFFMAN, P. G. 1987 The three-dimensional instability of strained vortices in a viscous fluid. Phys. Fluids 30, 2339-2342.

Leblanc, S. \& Cambon, C. 1997 On the three-dimensional instabilities of plane flows subjected to Coriolis force. Phys. Fluids 9, 1307-1316.

LEONARD, A., ROM-KeDAR, V.\& Wiggins, S. Fluid mixing and dynamical systems. Nuclear Physics B Proceedings Sisplements 2, 179-190.

Marshall, R. E. \& MYers, T. J. 1996 Wingtip generated wake vortices as radar targets. IEEE AES Systerns Magazine, December 1996, pp. 27-30.

Maxworthy, T. 1970 Turbulent vortex rings. J. Fluid Mech. 64, 227-239.

MILNE-Thomson, L.M. 1968 Theoretical Hydrodynamics, Macrillan.

Nespor, J., Hudson, E., Stegall, R. ANd Freedman, J. 1994 Doppler radar detection of vortex hazard inclicators. In NASA Conf. Proc. CP-10139, Part 2, pp. 651-688.

Noonkester, V. R. \& Richter, J. H. 1980 FM-CW radar sensing of the lower atmosphere. Radio $S$ ci. 15, 337-353. 
Ottersten, H. 1969 Atmospheric structure and radar backscattering in clear air. Radio Sci. 4, 1969, pp. 1179-1193.

Pedcosky, J. 1979 Geophysical Fluid Dynamics, Springer-Verlag.

Perry, R. B., Hinton, D. A., ANd Stuever. R. A. 1997 NASA wake vortex research for aircraft spacing. AIAA Paper 97-0057.

QเN, J.H. 1998 Numerical simulations of a turbulent axial vortex. PhD thesis, School of Aeronautics and Astronautics, Purdue Univ.

Revich, S. C. 1997 Accelerated destruction of aircraft wake vortices. PhD thesis, Dept. of Aero. \& Astro., Stanford Univ. Also Dept. of Aero. \& Astro. Report SUDAAR 705.

Reid, R. C., Prausnitz, J. M. \& Poling, B. E. 1987 The Properties of Gases and Liquids, McGraw-Hill.

Rom-Kedar, V., LEONARD, A. \& WiggiNs, S. 1990 An analytical study of transport, mixing and chaos in an unsteady flow. J. Fluid Mech. 214, 347-394.

Roтн, K.R. et al. 1989 The Kiernan Reentry Veasurements System on Kwajalein Atoll. The Lincoln Lab. J. 2, 247-276.

Silver, S. 1949 In Microwave antenna theory and design, McGraw-Hill.

SKoLNIK, M.I. (ed.) 1970 Radar Handbook, McGraw-Hill, 1970.

SPALART, P. R., 1996 On the motion of laminar wing wakes in a stratified fluid. J. Fluid Mech. 327, 139-60.

SPalart, P. R. 1998 Airplane trailing vortices. Ann. Rev. Fluid Mech. 30 107-38.

TATARSKI, V. I. 1961 Wave propagation in a turbulent medium. McGraw-Hill.

THAYER, G. D 1974 An improved equation for the radio refractive index of air. Radio $S c i .9$, 803-807.

Thomas, P. J. \& AUErbach, D. 1994 The observation of the simultaneous development of a long- and a short-wave instability mode on a vortex pair. J. Fluid Mech. 265, 289-302.

LeWEKE, T. \& WILliamson, C. H. K. 1998 Cooperative elliptic instability of a vortex pair. J. Fluid Mech. 360, 85-119.

Van Dyke, M. 1982 An Album of Fluid Motion, Parabolic Press.

VINCENT, R. A. S. et al. 1998 A VHF boundary layer radar: first results. Radio Sci. 33, 845-860. 